\title{
THE INTERVAL-VALUED INTUITIONISTIC FUZZY GEOMETRIC CHOQUET AGGREGATION OPERATOR BASED ON THE GENERALIZED BANZHAF INDEX AND 2-ADDITIVE MEASURE
}

\author{
Fanyong $\mathrm{MENG}^{\mathrm{a}}$, , Qiang $\mathrm{ZHANG}^{\mathrm{c}}$, Jiaquan $\mathrm{ZHAN}^{\mathrm{b}}$ \\ a School of Business, Central South University, Changsha, 410083 Hunan, China \\ ${ }^{\mathrm{b}}$ School of Management, Qingdao Technological University, Qingdao, 266520 Shandong, China \\ 'School of Management and Economics, Beijing Institute of Technology, \\ 100081 Beijing, China
}

Received 03 June 2012; accepted 01 December 2012

\begin{abstract}
Based on the operational laws on interval-valued intuitionistic fuzzy sets, the generalized Banzhaf interval-valued intuitionistic fuzzy geometric Choquet (GBIVIFGC) operator is proposed, which is also an interval-valued intuitionistic fuzzy value. It is worth pointing out that the GBIVIFGC operator can be seen as an extension of some geometric mean operators. Since the fuzzy measure is defined on the power set, it makes the problem exponentially complex. In order to overall reflect the interaction among elements and reduce the complexity of solving a fuzzy measure, we further introduce the GBIVIFGC operator w.r.t. 2-additive measures. Furthermore, if the information about weights of experts and attributes is incompletely known, the models of obtaining the optimal 2 -additive measures on criteria set and expert set are given by using the introduced cross entropy measure and the Banzhaf index. Finally, an approach to pattern recognition and multi-criteria group decision making under interval-valued intuitionistic fuzzy environment is developed, respectively.
\end{abstract}

Keywords: interval-valued intuitionistic fuzzy set, generalized Banzhaf index, Choquet integral, 2-additive measure.

JEL Classification: A14, C02, C61, C71.

\section{Introduction}

\section{Research background}

Since Zadeh (1965) first proposed fuzzy set (FS) theory, it has been widely investigated and applied to a variety of fields. However, a fuzzy set only gives us the membership information of an element. Later, Atanassov (1986) proposed the concept of intuitionistic fuzzy set (IFS),

Corresponding author Fanyong Meng

E-mail: mengfanyongtjie@163.com 
which is characterized by a degree of membership and a degree of non-membership. The sum of the membership degree and the non-membership degree of each ordered pair is less than or equal to one. The IFSs give us the possibility to model hesitation and uncertainty by using an additional degree (Wang 2009; Yager 2009). Atanassov $(1986,1999)$ further gave some basic operations and relations of IFSs. Xu (2007a) and Xu and Yager (2006) introduced some aggregation operators. The research results have been achieved in many different fields (Atanassov 1999; Hung, Yang 2008; Jiang et al. 2009; Bustince et al. 2007; Xu et al. 2008; Chen, Li 2010; Xu, Cai 2010a; De et al. 2001). However, in many real life situations, the exact values of the membership and non-membership would not be identified easily due to insufficiency in information availability. In 1989, Atanassov and Gargov (1989) introduced the concept of interval-valued intuitionistic fuzzy sets, which are characterized by an interval membership function and an interval non-membership function. Such a generalization further facilitates effectively representing inherent imprecision and uncertainty in the human decision making process. The application of interval-valued intuitionistic fuzzy sets for solving various decision making problems has received considerable attention recently (Atanassov 1994; Chen et al. 2011; Li 2010, 2011a, 2011b; Nayagam, Sivaraman 2011; Park et al. 2011; Xu, Chen 2007; Xu, Cai 2010a; Ye 2010; Yue 2011).

All above mentioned researches only considered situations where all elements in a set are independent, i.e., they only considered the addition of the importance of individual elements. However, in many practical situations, the elements are usually correlative (Grabisch 1995, 1996). As a powerful tool for measuring the interaction phenomenon among elements, fuzzy measures (Sugeno 1974) have been successfully used in decision problems (Labreuche, Grabisch 2003; Grabisch, Labreuche 2008; Yager 2003; Xu 2010; Tan, Chen 2010a, 2010b, 2011; Tan 2011a, 2011b; Tan et al. 2011; Zhang et al. 2011). Since the fuzzy measure is defined on the power set, it makes the problem exponentially complex. In order to deal with this issue, some special fuzzy measures were proposed, such as $\lambda$ - fuzzy measure (Sugeno 1974), $k$-additive measure (Grabisch 1997) and p-symmetric fuzzy measure (Miranda et al. 2002). Corresponding to fuzzy measures, fuzzy integrals as a useful tool for aggregating fuzzy information are also successfully used in many different fields (Grabisch et al. 2000).

\section{Research objectives}

Although many aggregation operators have been developed under intuitionistic fuzzy environment, there are some problems that should be pointed out: (1) The existing aggregation operators based on the Choquet integral do not consider the interaction among elements globally; (2) How to obtain the fuzzy measure on a set is not studied; (3) The complexity of solving a fuzzy measure is not taken into account.. In order to deal with these problems, the rest of this paper is organized as follows:

In section 1, some basic notations and conceptions are reviewed, which will be used in the following. In section 2, we introduce an aggregation operator for IVIFSs by using the Choquet integral and the generalized Banzhaf index, which is named as the generalized Banzhaf interval- valued intuitionistic fuzzy geometric Choquet (GBIVIFGC) operator. In order to simplify the complexity of solving a fuzzy measure, we further define the GBIVIFGC operator w.r.t. 2-adttitive measure, which is simply expressed as the GABIVIFGC operator. 
In section 3, we first give a new cross entropy measure for IVIFSs. Then, the models of getting the optimal 2-additive measures on criteria set and expert set are given, respectively. In section 4 , an approach to pattern recognition and multi-criteria group decision making under interval-valued intuitionistic fuzzy environment is respectively developed. Meantime, the corresponding examples are given to illustrate the concrete application of the introduced methods. The conclusions are made in the last section.

\section{Preliminaries}

In this section, we will review some basic notations and definitions of interval-valued intuitionistic fuzzy sets, fuzzy measures and the Choquet integral, which will be used in the following.

\subsection{Interval-valued intuitionistic fuzzy sets}

Definition 1 (Atanassov, Gargov 1989). Let $X$ be a no empty finite set. An IVIFS $A$ in $X$ is expressed as:

$$
A=\left\{\left\langle x,\left[\mu_{l}(x), \mu_{u}(x)\right],\left[v_{l}(x), v_{u}(x)\right]\right\rangle \mid x \in X\right\}
$$

where $\left[\mu_{l}(x), \mu_{u}(x)\right] \subseteq[0,1]$ and $\left[v_{l}(x), v_{u}(x)\right] \subseteq[0,1]$ respectively denote the intervalvalued degrees of membership and non-membership of element $x \in X$ with the condition $\mu_{u}(x)+v_{u}(x) \leq 1$.

For any $x \in X,\left[\pi_{l}(x), \pi_{u}(x)\right]=\left[1-\mu_{u}(x)-v_{u}(x), 1-\mu_{l}(x)-v_{l}(x)\right]$ is the interval hesitance degree of element $x$ to $A$. $\left[\pi_{l}(x), \pi_{u}(x)\right]$ is also called interval intuitionistic index. When $\pi_{l}(x)=\pi_{u}(x)$ for all $x \in X$, the IVIFS $A$ degenerates to be an IFS. Especially, when $\pi_{l}(x)=\pi_{u}(x)=0$ for all $x \in X$, the IVIFS $A$ degenerates to be a fuzzy set.

In order to denote simply, any interval-valued intuitionistic fuzzy value (IVIFV) $\tilde{\alpha}$ can be expressed by $\tilde{\alpha}=([a, b],[c, d])$, where $[a, b] \subseteq[0,1]$ and $[c, d] \subseteq[0,1]$ respectively denote the interval-valued degrees of membership and non-membership with the condition $b+d \leq 1$ (see Xu, Chen 2007). Let $\Omega$ be the set of all IVIFVs on $X$. Obviously, we know that $\tilde{\alpha}^{+}=([1,1],[0,0])$ and $\tilde{\alpha}^{-}=([0,0],[1,1])$ are the largest and smallest IVIFVs, respectively (see Xu 2007b).

Combine the interval intuitionistic index, $\mathrm{Xu}$ (2008) introduced the following form to express an IVIFS $A=\left\{\left\langle x,\left[\mu_{l}(x), \mu_{u}(x)\right],\left[v_{l}(x), v_{u}(x)\right],\left[\pi_{l}(x), \pi_{u}(x)\right]\right\rangle \mid x \in X\right\}$. Thus, any IVIFV $\tilde{\alpha}=([a, b],[c, d])$ can be expressed by $\tilde{\alpha}=([a, b],[c, d],[e, f])$ with $e=1-b-d$ and $f=1-c-a$. In this situation, it is clear that $\tilde{\alpha}^{+}=([1,1],[0,0],[0,0])$ and $\tilde{\alpha}^{-}=([0,0],[1,1],[0,0])$ are the largest and smallest IVIFVs, respectively (see Xu 2008).

Definition 2 (Xu 2007b). Let $\tilde{\alpha}=\left(\left[a_{1}, b_{1}\right],\left[c_{1}, d_{1}\right]\right)$ and $\tilde{\gamma}=\left(\left[a_{2}, b_{2}\right],\left[c_{2}, d_{2}\right]\right)$ be any two IVIFVs in $\Omega$, then some operations of $\tilde{\alpha}$ and $\tilde{\gamma}$ are defined by:
1) $\tilde{\alpha} \otimes \tilde{\gamma}=\left(\left[a_{1} a_{2}, b_{1} b_{2}\right],\left[c_{1}+c_{2}-c_{1} c_{2}, d_{1}+d_{2}-d_{1} d_{2}\right]\right)$,
2) $\tilde{\alpha}^{\lambda}=\left(\left[a_{1}^{\lambda}, b_{1}^{\lambda}\right],\left[1-\left(1-c_{1}\right)^{\lambda}, 1-\left(1-d_{1}\right)^{\lambda}\right]\right), \lambda>0$. 
Proposition 1 (Xu 2007b). Let $\tilde{\alpha}=\left(\left[a_{1}, b_{1}\right],\left[c_{1}, d_{1}\right]\right)$ and $\tilde{\gamma}=\left(\left[a_{2}, b_{2}\right],\left[c_{2}, d_{2}\right]\right)$ be any two IVIFVs in $\Omega$, then:

1) $\tilde{\alpha} \otimes \tilde{\gamma}=\tilde{\gamma} \otimes \tilde{\alpha}$,

2) $(\tilde{\alpha} \otimes \tilde{\gamma})^{\lambda}=\tilde{\alpha}^{\lambda} \otimes \tilde{\gamma}^{\lambda} \quad \forall \lambda>0$,

3) $\tilde{\alpha}^{\lambda_{1}+\lambda_{2}}=\tilde{\alpha}^{\lambda_{1}} \otimes \tilde{\alpha}^{\lambda_{2}} \quad \forall \lambda_{1}, \lambda_{2}>0$.

Definition 3. Let $\tilde{\alpha}=\left(\left[a_{1}, b_{1}\right],\left[c_{1}, d_{1}\right]\right)$ and $\tilde{\gamma}=\left(\left[a_{2}, b_{2}\right],\left[c_{2}, d_{2}\right]\right)$ be any two IVIFVs in $\Omega$, then:

1) $\tilde{\alpha} \leq \tilde{\gamma}$ if and only if $a_{1} \leq a_{2}, b_{1} \leq b_{2}, c_{1} \geq c_{2}$ and $d_{1} \geq d_{2}$,

2) $\tilde{\alpha}=\tilde{\gamma}$ if and only if $\tilde{\alpha} \leq \tilde{\gamma}$ and $\tilde{\alpha} \geq \tilde{\gamma}$,

3) $\tilde{\alpha}^{C}=\left(\left[c_{1}, d_{1}\right],\left[a_{1}, b_{1}\right]\right)$.

\subsection{Fuzzy measures and the Choquet integral}

In some real decision problems, it is not suitable to measure the importance of criteria by using additive measures because the independence of them is usually violated. In 1974, Sugeno (1974) introduced the concept of fuzzy measure, which is a powerful tool for modeling interaction among elements (Grabisch, Roubens 1999; Kojadinovic 2003, 2005) and dealing with decision problems (Grabisch 1995, 1996; Labreuche, Grabisch 2003; Grabisch, Labreuche 2008; Xu 2010; Tan, Chen 2010a, 2010b).

Definition 4 (Sugeno 1974). A fuzzy measure $\mu$ on finite set $N$ is a set function $\mu: P(N) \rightarrow[0,1]$ satisfying:

1) $\mu(\varnothing)=0, \mu(N)=1$,

2) $A \subseteq B$ implies $\mu(A) \leq \mu(B)$,

where $P(N)$ is the power set of $N$.

In the multi-criteria group decision making, $\mu(A)$ can be viewed as the importance of the criteria set or expert set $A$. As a result, in addition to the usual weights on criteria set or expert set taken separately, weights on any combination of criteria set or expert set are also defined.

From Definition 4, we know the fuzzy measure is defined on the power set, which makes the problem exponentially complex. Thus, it is not easy to get the fuzzy measure of each combination in a set when it is large. In order to reflect the interaction among elements and simplify the complexity of solving a fuzzy measure, some special fuzzy measures were introduced (Sugeno 1974; Grabisch 1997; Miranda et al. 2002).

Let $f:\{0,1\} \rightarrow \mathbb{R}$. be a pseudo-Boolean function. Grabisch (1997) pointed out that any fuzzy measure $\mu$ can be seen as a particular case of pseudo-Boolean function, and put under a multilinear polynomial in $n$ variables:

$$
\mu(A)=\sum_{T \subseteq N}\left[a_{T} \prod_{i \in T} y_{i}\right] \forall A \subseteq N
$$

where $a_{T} \in \mathbb{R}, y=\left(y_{1}, y_{2}, \ldots, y_{n}\right) \in\{0,1\}^{n}$, and $y_{i}=1$ iff $i \in A$. 
The set of coefficients $a_{T}(T \subseteq N)$ in fact corresponds to the Möbius transform, denoted by $a_{T}=\sum_{S \subseteq T}(-1)^{|T \backslash S|} \mu(S)$. Since the transform is inversible, $\mu$ can be recovered from $a_{T}$ by $\mu(A)=\sum_{B \subseteq A} a_{B}$

Definition 5 (Grabisch 1997). A fuzzy measure $\mu$ on $N$ is said to be $k$-additive if its corresponding pseudo-Boolean function is a multilinear polynomial of degree $k$, i.e., $a_{T}=0$ for all $T$ such that $|T|>k$, and there exists at least one subset $T$ with $k$ elements such that $a_{T} \neq 0$.

When $k=1$, the $k$-additive measure $\mu$ reduces to be an additive measure; When $k=n$, the $k$-additive $\mu$ is a fuzzy measure as usual. Especially, when $k=2$, by Eq. (1) we get a 2 -additive measure. For a 2 -additive measure $\mu$, one can easily get (Grabisch 1997), for any $S \subseteq N$ with $|S| \geq 2$ :

$$
\mu(S)=\sum_{i=1}^{n} a_{i} x_{i}+\sum_{\{i, j\} \subseteq N} a_{i j} x_{i} x_{j}=\sum_{i \in S} a_{i}+\sum_{\{i, j\} \subseteq S} a_{i j}=\sum_{\{i, j\} \subseteq S} \mu(i, j)-(|S|-2) \sum_{i \in S} \mu(i),
$$

where $\mu(i)=a_{i}, \mu(i, j)=a_{i}+a_{j}+a_{i j}$.

For a 2 -additive measure, we only need $n(n+1) / 2$ coefficients to determine it for a set with $n$ elements.

Theorem 1 (Grabisch 1997). Let $\mu$ be a fuzzy measure on $N$, then $\mu$ is a 2 -additive measure if and only if there exist coefficients $\mu(i)$ and $\mu(i, j)$ for all $i, j \in N$ that satisfy the following conditions:

1) $\mu(i) \geq 0 \quad \forall i \in N$,

2) $\sum_{\{i, j\} \subseteq N} \mu(i, j)-(|N|-2) \sum_{i \in N} \mu(i)=1$,

3) $\sum_{i \subseteq S \backslash k}(\mu(i, k)-\mu(i)) \geq(|S|-2) \mu(k) \quad \forall S \in N$ s.t. $k \in S$ and $|S| \geq 2$.

Corresponding to fuzzy measures, fuzzy integrals are important aggregation operators w.r.t. fuzzy measures, which are studied by many researchers (Sugeno 1974; Grabisch 1997; Miranda et al. 2002; Dubois, Prade 1988). One of the most important fuzzy integrals is the Choquet integral (Choquet 1953). Yager (2003) introduced the Choquet aggregation operator on fuzzy sets. Xu (2010) and Tan (2011b) studied some Choquet aggregation operators on IFSs and IVIFSs. The application of the Choquet integral has received considerable attention recently (Labreuche, Grabisch 2003; Grabisch, Labreuche 2008; Yager 2003; Xu 2010; Tan 2011a, 2011b). In 1996, Grabisch (1996) put forward the following concept of the Choquet integral on discrete sets.

Definition 6. Let $f$ be a positive real-valued function on $X=\left\{x_{1}, x_{2}, \ldots, x_{n}\right\}$, and $\mu$ be a fuzzy measure on $N$. The discrete Choquet integral of $f$ w.r.t. $\mu$ is defined by:

$$
C_{\mu}\left(f\left(x_{(1)}\right), f\left(x_{(2)}\right), \ldots, f\left(x_{(n)}\right)\right)=\sum_{i=1}^{n} f\left(x_{(i)}\right)\left(\mu\left(A_{(i)}\right)-\mu\left(A_{(i+1)}\right)\right),
$$

where $(\cdot)$ indicates a permutation on $N$ such that $f\left(x_{(1)}\right) \leq f\left(x_{(2)}\right) \leq \ldots \leq f\left(x_{(n)}\right)$, and $A_{(i)}=\{i, \ldots, n\}$ with $A_{(n+1)}=\varnothing$. 
Based on the Choquet integral, $\mathrm{Xu}$ (2010) defined the following interval-valued intuitionistic fuzzy correlated averaging (IVIFCA) operator:

$$
\operatorname{IVIFCA}\left(\tilde{\alpha}_{1}, \tilde{\alpha}_{2}, \ldots, \tilde{\alpha}_{n}\right)=\bigoplus_{i=1}^{n} \tilde{\alpha}_{(i)}\left(\mu\left(A_{(i)}\right)-\mu\left(A_{(i+1)}\right)\right),
$$

and $\mathrm{Xu}(2010)$ and Tan (2011b) respectively presented the following interval-valued intuitionistic fuzzy geometric aggregation (IVIFGA) operator:

$$
\operatorname{IVIFGA}\left(\tilde{\alpha}_{1}, \tilde{\alpha}_{2}, \ldots, \tilde{\alpha}_{n}\right)=\bigotimes_{i=1}^{n} \tilde{\alpha}_{(i)} \mu\left(A_{(i)}\right)-\mu\left(A_{(i+1)}\right),
$$

where $(\cdot)$ indicates a permutation on $X$ such that $\tilde{\alpha}_{(1)} \leq \tilde{\alpha}_{(2)} \leq \ldots . \leq \tilde{\alpha}_{(n)}$, and $A_{(i)}=\{i, \ldots, n\}$ with $A_{(n+1)}=\varnothing, \boldsymbol{m}$ is the fuzzy measure on index set $N=\{1,2, \ldots, n\}$.

Although the IVIFCA and IVIFGA operators can indict the importance of any combination in a set, they can not overall reflect the interaction among all combinations $A_{(i)}(i=1,2, \ldots, n)$ as it only considers the interaction between subsets $A_{(i)}$ and $A_{(i+1)}(i=1,2, \ldots, n)$.

\section{The generalized Banzhaf interval-valued intuitionistic fuzzy geometric Choquet operator}

\subsection{The general case}

In order to measure the power or the strength of each coalition in a game rather than that of each player, Marichal (2000) gave an expectation index called the generalized Banzhaf index in game theory, which is expressed by:

$$
\varphi_{S}(\mu, N)=\sum_{T \subseteq N \backslash S} \frac{1}{2^{|N|-|S|}}(\mu(S \bigcup T)-\mu(T)) \quad \forall S \subseteq N .
$$

From Eq. (3), when $|S|=1$, suppose $S=\{i\}$, then we get the Banzhaf function (Banzhaf 1965) as follows:

$$
\varphi_{i}(\mu, N)=\sum_{T \subseteq N \backslash i} \frac{1}{2^{|N|-1}}(\mu(i \bigcup T)-\mu(T)) \quad \forall i \in N .
$$

When we apply Eq. (3) in the setting of multi-criteria decision making. $\varphi_{S}(\mu, N)$ can be viewed as the importance of the criteria set $S$. For any $S \subseteq N$, if there is no interaction between the coalition $S \subseteq N$ and any coalition in $N \backslash S$, then $\mu$ degenerates to be an additive measure. Namely, $\varphi_{S}(\mu, N)=\sum_{i \in S} \varphi_{i}(\mu, N)=\sum_{i \in S} \mu(i)$ for any $S \subseteq N$.

Theorem 1. Let $\mu: P(N) \rightarrow[0,1]$ be a fuzzy measure on $N$, then the generalized Banzhaf index $\varphi$ given as Eq. (3) is also a fuzzy measure.

Proof. By Eq. (3), it is obvious that $\varphi_{\varnothing}(\mu, N)=0$ and $\varphi_{N}(\mu, N)=1$. 
For any $S, K \subseteq N$ with $S \subseteq K$, when $|S|+1=|K|$, without loss of generality, suppose $S \bigcup i=K$. From Eqs (3) and (4), we get:

$$
\begin{aligned}
\varphi_{S}(\mu, N)= & \sum_{T \subseteq N \backslash S} \frac{1}{2^{|N|-|S|}}(\mu(S \cup T)-\mu(T))= \\
& \sum_{T \subseteq N \backslash S \cup i} \frac{1}{2^{|N|-|S|}}(\mu(S \cup T)-\mu(T))+\sum_{T \subseteq N \backslash S \cup i} \frac{1}{2^{|N|-|S|-1}}(\mu(S \cup T \bigcup i)-\mu(T \bigcup i))
\end{aligned}
$$

and

$$
\begin{aligned}
\varphi_{K}(\mu, N)= & \sum_{T \subseteq N \backslash K} \frac{1}{2^{|N|-|K|}}(\mu(K \cup T)-\mu(T))= \\
& \sum_{T \subseteq N \backslash S \cup i} \frac{1}{2^{|N|-|S|-1}}(\mu(S \bigcup T \bigcup i)-\mu(T)) .
\end{aligned}
$$

According to the monotonicity of $\mu$, it is clear that $\mu(T) \leq \mu(T \bigcup i)$ and $\mu(S \cup T) \leq \mu(S \cup T \bigcup i)$. Thus:

$$
\varphi_{S}(\mu, N) \leq \varphi_{K}(\mu, N)
$$

When $|S|+p=|K|$, without loss of generality, suppose $S \cup\left\{i_{i}, i_{2}, \ldots, i_{p}\right\}=K$ and let $S_{1}=S \bigcup\left\{i_{1}\right\}, S_{2}=S_{1} \bigcup\left\{i_{2}\right\}, \ldots, S_{p}=S_{p-1} \bigcup\left\{i_{p}\right\}$. From above, we obtain:

$$
\varphi_{S}(\mu, N) \leq \varphi_{S_{1}}(\mu, N) \leq \ldots . \varphi_{S_{p}}(\mu, N)=\varphi_{K}(\mu, N) .
$$

From Definition 4, we know that $\varphi$ given as Eq. (3) is a fuzzy measure.

From Definition 6, we define the generalized Banzhaf geometric Choquet integral as follows:

$$
C_{\varphi(\mu, N)}\left(f\left(x_{(1)}\right), f\left(x_{(2)}\right), \ldots, f\left(x_{(n)}\right)\right)=\bigotimes_{i=1}^{n} f\left(x_{(i)}\right)^{\varphi_{A_{(i)}}(\mu, N)-\varphi_{A_{(i+1)}}(\mu, N)}
$$

where (.) indicates a permutation on $N$ such that $f\left(x_{(1)}\right) \leq f\left(x_{(2)}\right) \leq \ldots \leq f\left(x_{(n)}\right)$, and $A_{(i)}=\{i, \ldots, n\}$ with $A_{(n+1)}=\varnothing$.

Definition 7. Let $\tilde{\alpha}_{i}=\left(\left[a_{i}, b_{i}\right],\left[c_{i}, d_{i}\right]\right)(i=1,2, \ldots, n)$ be a collection of IVIFVs in $\Omega$, and $\mu$ be a fuzzy measure on $N$. A GBIVIFGC operator of $\tilde{\alpha}_{i}$ is defined by:

$$
(C B) \int \tilde{\alpha} d \varphi(\mu, N)=\operatorname{GBIVIFGC}\left(\tilde{\alpha}_{1}, \tilde{\alpha}_{2}, \ldots, \tilde{\alpha}_{n}\right)=\otimes_{i=1}^{n}\left(\tilde{\alpha}_{(i)}\right)^{\varphi_{A_{(i)}}(\mu, N)-\varphi_{A_{(i+1)}}(\mu, N)},
$$

where $(\cdot)$ indicates a permutation on $N$ such that $\tilde{\alpha}_{(1)} \leq \tilde{\alpha}_{(2)} \leq \ldots \leq \tilde{\alpha}_{(n)}$, and $A_{(i)}=\{i, \ldots, n\}$ with $A_{(n+1)}=\varnothing$.

Remark 1. When we restrict the domain of IVIFSs in the setting of IFSs, then Eq. (5) degenerates to be the generalized Banzhaf intuitionistic fuzzy geometric Choquet (GBIFGC) operator. 
Theorem 2. Let $\tilde{\alpha}_{i}=\left(\left[a_{i}, b_{i}\right],\left[c_{i}, d_{i}\right]\right)(i=1,2, \ldots, n)$ be a collection of IVIFVs in $\Omega$, and $\mu$ be a fuzzy measure on $N$. Then their aggregated value by using the GBIVIFGC operator is also an IVIFV, and denoted by:

$$
\begin{aligned}
& \operatorname{GBIVIFGC}\left(\tilde{\alpha}_{1}, \tilde{\alpha}_{2}, \ldots, \tilde{\alpha}_{n}\right)=\left(\left[\prod_{i=1}^{n} a_{(i)} \varphi_{A_{(i)}}(\mu, N)-\varphi_{A_{(i+1)}}(\mu, N), \prod_{i=1}^{n} b_{(i)} \varphi_{A_{(i)}}(\mu, N)-\varphi_{A_{(i+1)}}(\mu, N)\right],\right. \\
& {\left[1-\prod_{i=1}^{n}\left(1-c_{(i)}\right)^{\varphi_{A_{(i)}}(\mu, N)-\varphi_{A_{(i+1)}}(\mu, N)}, 1-\prod_{i=1}^{n}\left(1-d_{(i)}\right)^{\left.\left.\varphi_{A_{(i)}}(\mu, N)-\varphi_{A_{(i+1)}}(\mu, N)\right]\right),}\right.}
\end{aligned}
$$

where $(\cdot)$ indicates a permutation on $N$ such tha $\tilde{\alpha}_{(1)} \leq \tilde{\alpha}_{(2)} \leq \ldots \leq \tilde{\alpha}_{(n)}$, and $A_{(i)}=\{i, \ldots, n\}$ with $A_{(n+1)}=\varnothing$.

Proof. The first result follows quickly from Definition 1. Below we prove Eq. (6) by using mathematical induction on $n$.

(i) When $n=2$, from Theorem 1, we have:

$$
\begin{array}{r}
\left(\tilde{\alpha}_{(1)}\right)^{\varphi_{A_{(1)}}(\mu, N)-\varphi_{A_{(2)}}(\mu, N)}=\left(\left[a_{(1)}{ }^{\varphi_{A_{(1)}}(\mu, N)-\varphi_{A_{(2)}}(\mu, N)}, b_{(1)}{ }^{\left.\varphi_{A_{(1}}(\mu, N)-\varphi_{A_{(2)}}(\mu, N)\right]}\right.\right. \\
\left.\left[1-\left(1-c_{(1)}\right)^{\varphi_{A_{(1)}}(\mu, N)-\varphi_{A_{(2)}}(\mu, N)}, 1-\left(1-d_{(1)}\right)^{\varphi_{A_{(1)}}(\mu, N)-\varphi_{A_{(2)}}(\mu, N)}\right]\right)
\end{array}
$$

and

$$
\begin{array}{r}
\left(\tilde{\alpha}_{(2)}\right)^{\varphi_{A_{(2)}}(\mu, N)-\varphi_{A_{(3)}}(\mu, N)}=\left(\left[a_{(2)} \varphi_{A_{(2)}}(\mu, N)-\varphi_{A_{(3)}}(\mu, N), b_{(2)} \varphi_{A_{(2)}}^{\left.(\mu, N)-\varphi_{A_{(3)}}(\mu, N)\right],}\right.\right. \\
\left.\left[1-\left(1-c_{(2)}\right)^{\varphi_{A_{(2)}}(\mu, N)-\varphi_{A_{(3)}}(\mu, N)}, 1-\left(1-d_{(2)}\right)^{\varphi_{A_{(2)}}(\mu, N)-\varphi_{A_{(3)}}(\mu, N)}\right]\right) .
\end{array}
$$

Thus:

$$
\begin{aligned}
& \operatorname{GBIVIFGC}\left(\tilde{\alpha}_{1}, \tilde{\alpha}_{2}\right)=\left(\tilde{\alpha}_{(1)}\right)^{\varphi_{A_{(1)}}(\mu, N)-\varphi_{A_{(2)}}(\mu, N)} \otimes\left(\tilde{\alpha}_{(2)}\right)^{\varphi_{A_{(2)}}(\mu, N)-\varphi_{A_{(3)}}(\mu, N)}= \\
& \left(\left[a_{(1)}{ }^{\varphi_{A_{(1)}}}(\mu, N)-\varphi_{A_{(2)}}(\mu, N) a_{(2)} \varphi_{A_{(2)}}(\mu, N)-\varphi_{A_{(3)}}(\mu, N),\right.\right. \\
& \left.b_{(1)} \varphi_{A_{(1)}}(\mu, N)-\varphi_{A_{(2)}}(\mu, N) b_{(2)} \varphi_{A_{(2)}}(\mu, N)-\varphi_{A_{(3)}}(\mu, N)\right] \text {, }
\end{aligned}
$$

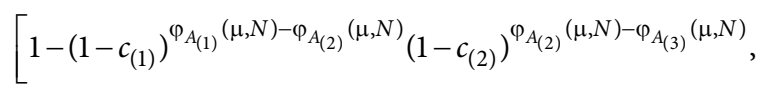

$$
\begin{aligned}
& \left.\left.1-\left(1-d_{(1)}\right)^{\varphi_{A_{(1)}}(\mu, N)-\varphi_{A_{(2)}}(\mu, N)}\left(1-d_{(2)}\right)^{\varphi_{A_{(2)}}(\mu, N)-\varphi_{A_{(3)}}(\mu, N)}\right]\right)= \\
& \left(\left[\prod_{i=1}^{2} a_{(i)} \varphi_{A_{(i)}}(\mu, N)-\varphi_{A_{(i+1)}}(\mu, N), \prod_{i=1}^{2} b_{(i)} \varphi_{A_{(i)}}(\mu, N)-\varphi_{A_{(i+1)}}(\mu, N)\right],\right. \\
& \left.\left[1-\prod_{i=1}^{2}\left(1-c_{(i)}\right)^{\varphi_{A_{(i)}}(\mu, N)-\varphi_{A_{(i+1)}}(\mu, N)}, 1-\prod_{i=1}^{2}\left(1-d_{(i)}\right)^{\varphi_{A_{(i)}}(\mu, N)-\varphi_{A_{(i+1)}}(\mu, N)}\right]\right) .
\end{aligned}
$$


(ii) Hypothesis, Eq. (6) holds for $n=k(k \geq 2))$, namely,

$\operatorname{GBIVIFGC}\left(\tilde{\alpha}_{1}, \tilde{\alpha}_{2}, \ldots, \tilde{\alpha}_{k}\right)=$

$$
\begin{aligned}
& \left(\left[\prod_{i=1}^{k} a_{(i)} \varphi_{A_{(i)}}(\mu, N)-\varphi_{A_{(i+1)}}(\mu, N), \prod_{i=1}^{k} b_{(i)} \varphi_{A_{(i)}}(\mu, N)-\varphi_{A_{(i+1)}}(\mu, N)\right],\right. \\
& {\left[1-\prod_{i=1}^{k}\left(1-c_{(i)}\right)^{\varphi_{A_{(i)}}(\mu, N)-\varphi_{A_{(i+1)}}(\mu, N)}, 1-\prod_{i=1}^{k}\left(1-d_{(i)}\right)^{\left.\left.\varphi_{A_{(i)}}(\mu, N)-\varphi_{A_{(i+1)}}(\mu, N)\right]\right) .}\right.}
\end{aligned}
$$

When $n=k+1$, by the operational laws given in Definition 2, we get:

$$
\begin{aligned}
& \operatorname{GBIVIFGC}\left(\tilde{\alpha}_{1}, \tilde{\alpha}_{2}, \ldots, \tilde{\alpha}_{k+1}\right)= \\
& \left(\left[\prod_{i=1}^{k} a_{(i)} \varphi_{A_{(i)}}(\mu, N)-\varphi_{A_{(i+1)}}(\mu, N), \prod_{i=1}^{k} b_{(i)} \varphi_{A_{(i)}}(\mu, N)-\varphi_{A_{(i+1)}}(\mu, N)\right],\right. \\
& \left.\left[1-\prod_{i=1}^{k}\left(1-c_{(i)}\right)^{\varphi_{A_{(i)}}(\mu, N)-\varphi_{A_{(i+1)}}(\mu, N)}, 1-\prod_{i=1}^{k}\left(1-d_{(i)}\right)^{\varphi_{A_{(i)}}(\mu, N)-\varphi_{A_{(i+1)}}(\mu, N)}\right]\right) \otimes \\
& \left(\left[a_{(k+1)} \varphi_{A_{(k+1)}}^{(\mu, N)-\varphi_{A_{(k+2)}}(\mu, N)}, b_{(k+1)} \varphi_{A_{(k+1)}}(\mu, N)-\varphi_{A_{(k+2)}}(\mu, N)\right],\right. \\
& \left.\left[1-\left(1-c_{(k+1)}\right) \varphi^{\varphi_{A_{(k+1)}}(\mu, N)-\varphi_{A_{(k+2)}}(\mu, N)}, 1-\prod_{i=1}^{k}\left(1-d_{(k+1)}\right)^{\varphi_{A_{(k+1)}}(\mu, N)-\varphi_{A_{(k+2)}}(\mu, N)}\right]\right)= \\
& \left(\left[\prod_{i=1}^{k+1} a_{(i)} \varphi_{A_{(i)}}(\mu, N)-\varphi_{A_{(i+1)}}(\mu, N), \prod_{i=1}^{k+1} b_{(i)} \varphi_{A_{(i)}}(\mu, N)-\varphi_{A_{(i+1)}}(\mu, N)\right],\right. \\
& \left.\left[1-\prod_{i=1}^{k+1}\left(1-c_{(i)}\right)^{\varphi_{A_{(i)}}(\mu, N)-\varphi_{A_{(i+1)}}(\mu, N)}, 1-\prod_{i=1}^{k+1}\left(1-d_{(i)}\right)^{\varphi_{A_{(i)}}(\mu, N)-\varphi_{A_{(i+1)}}(\mu, N)}\right]\right) .
\end{aligned}
$$

That is, for $n=k+1$, the Eq. (6) still holds, and the result is obtained.

From Theorem 2, it is not difficult to know the GBIVIFGC operator satisfies the properties of commutativity, idempotency, comonotonicity and boundary, which are important to an aggregation operator.

\subsection{The GBIVIFGC operator w.r.t. 2-additive measure}

In this section, we shall research the GABIVIFGC operator, which not only globally reflects the interaction among elements, but also greatly simplifies the complexity of solving a fuzzy measure.

Theorem 3. Let $\mu$ be a 2-fuzzy measure on $N$, then the generalized Banzhaf index $\varphi$ for $\mu$ can be expressed by:

$$
\varphi_{S}(\mu, N)=\sum_{\{i, j\} \subseteq S} \mu(i, j)+\frac{1}{2} \sum_{i \in S, j \in N \backslash S}(\mu(i, j)-|S| \mu(j))-\frac{|N|+|S|-4}{2} \sum_{i \in S} \mu(i),
$$

for any $S \subseteq N$ with $|S| \geq 2$, and when $|S|=1$, 


$$
\varphi_{i}(\mu, N)=\frac{3-|N|}{2} \mu(i)+\frac{1}{2} \sum_{j \in N \backslash i}(\mu(i, j)-\mu(j)),
$$

for any $\{i\}=S \subseteq N$.

Proof. For Eq. (7): By Eqs (2) and (3), we get:

$$
\begin{aligned}
& \varphi_{S}(\mu, N)=\sum_{T \subseteq N \backslash S} \frac{1}{2^{|N|-|S|}}(\mu(S \bigcup T)-\mu(T))= \\
& \sum_{T \subseteq N \backslash S} \frac{1}{2^{|N|-|S|}}\left(\left(\sum_{\{i, j\} \subseteq S \cup T} \mu(i, j)-(|S|+|T|-2) \sum_{i \in S \cup T} \mu(i)\right)-\right. \\
& \left.\left(\sum_{\{i, j\} \subseteq T} \mu(i, j)-(|T|-2) \sum_{i \in T} \mu(i)\right)\right)= \\
& \sum_{T \subseteq N \backslash S} \frac{1}{2^{|N|-|S|}}\left(\sum_{\{i, j\} \subseteq S} \mu(i, j)+\sum_{i \subseteq S, j \in T} \mu(i, j)-(|S|+|T|-2) \sum_{i \in S} \mu(i)-|S| \sum_{j \in T} \mu(j)\right)= \\
& \sum_{T \subseteq N \backslash S} \frac{1}{2^{|N|-|S|}} \sum_{\{i, j\} \subseteq S} \mu(i, j)-\sum_{T \subseteq N \backslash S} \frac{|S|}{2^{|N|-|S|}} \sum_{j \in T} \mu(j)+\sum_{T \subseteq N \backslash S} \frac{1}{2^{|N|-|S|}} \sum_{i \subseteq S, j \in T} \mu(i, j)- \\
& \sum_{T \subseteq N \backslash S} \frac{(|S|+|T|-2)}{2^{|N|-|S|}} \sum_{i \in S} \mu(i)= \\
& \sum_{\{i, j\} \subseteq S} \frac{\sum_{|T|=0}^{|N|-|S|} C_{|N|-|S|}^{|T|}}{2^{|N|-|S|}} \mu(i, j)+\sum_{i \subseteq S, j \in N \backslash S} \frac{\sum_{|T|=0}^{|N|-|S|-1} C_{|N|-|S|-1}^{|T|}}{2^{|N|-|S|}} \mu(i, j)-\sum_{j \in N \backslash S} \frac{|S| \sum_{|T|=0}^{|N|-|S|-1} C_{|N|-|S|-1}^{|T|}}{2^{|N|-|S|}} \mu(j)- \\
& \sum_{i \in S} \frac{\sum_{|T|=0}^{|N|-|S|}|T| C_{|N|-|S|}^{|T|}+(|S|-2) \sum_{|T|=0}^{|N|-|S|} C_{|N|-|S|}^{|T|}}{2^{|N|-|S|}} \mu(i)= \\
& \sum_{\{i, j\} \subseteq S} \frac{2^{|N|-|S|}}{2^{|N|-|S|}} \mu(i, j)+\sum_{i \subseteq S, j \in N \backslash S} \frac{2^{|N|-|S|-1}}{2^{|N|-|S|}} \mu(i, j)-\sum_{j \in N \backslash S} \frac{|S| 2^{|N|-|S|-1}}{2^{|N|-|S|}} \mu(j)- \\
& \sum_{i \in S} \frac{(|N|-|S|) 2^{|N|-|S|-1}+(|S|-2) 2^{|N|-|S|}}{2^{|N|-|S|}} \mu(i)= \\
& \sum_{\{i, j\} \subseteq S} \mu(i, j)+\sum_{i \subseteq S, j \in N \backslash S} \frac{1}{2} \mu(i, j)-\sum_{j \in N \backslash S} \frac{|S|}{2} \mu(j)-\sum_{i \in S} \frac{|N|+|S|-4}{2} \mu(i)= \\
& \sum_{\{i, j\} \subseteq S} \mu(i, j)+\frac{1}{2} \sum_{i \in S, j \in N \backslash S}(\mu(i, j)-|S| \mu(j))-\frac{|N|+|S|-4}{2} \sum_{i \in S} \mu(i) .
\end{aligned}
$$


For Eq. (8): Similar to Eq. (7), we have:

$$
\begin{aligned}
\varphi_{i}(\mu, N)= & \sum_{T \subseteq N \backslash i} \frac{1}{2^{|N|-1}}(\mu(i \cup T)-\mu(T))= \\
& \frac{1}{2^{|N|-1}} \mu(i)+\frac{1}{2^{|N|-1}} \sum_{T \subseteq N \backslash i}\left(\sum_{j \in T}(\mu(i, j)-\mu(j))-(|T|-1) \mu(i)\right)= \\
& \frac{1}{2^{|N|-1}} \mu(i)+\sum_{j \in N \backslash i} \frac{|T|=0}{2^{|N|-1}}(\mu(i, j)-\mu(j))-\sum_{|T|=1}^{|N|-2} \frac{|T|-1) C_{|N|-1}^{|T|} \mu(i)=}{2^{|N|-1}} \\
& \frac{1}{2^{|N|-1}} \mu(i)+\sum_{j \in N \backslash i} \frac{2^{|N|-2}}{2^{|N|-1}}(\mu(i, j)-\mu(j))-\frac{(|N|-1) 2^{|N|-2}-2^{|N|-1}+1}{2^{|N|-1}} \mu(i)= \\
& \frac{3-|N|}{2} \mu(i)+\frac{1}{2} \sum_{j \in N \backslash i}(\mu(i, j)-\mu(j)) .
\end{aligned}
$$

Theorem 4. Let $\mu$ be a 2-fuzzy measure on $N$, and $\varphi$ be the generalized Banzhaf index for $\mu$ given as Eq. (3). Then,

$$
\varphi_{S \cup i}(\mu, N)-\varphi_{S}(\mu, N)=\varphi_{i}(\mu, N)
$$

for any $i \in N$ and any $S \subseteq N$ with $i \notin S$.

Proof. When $S=\varnothing$, the result obviously holds. When $|S|=1$, without loss of generality, suppose $S=\{l\}$, and then from Theorem 3, we get:

$$
\varphi_{\{i, l\}}(\mu, N)=\mu(i, l)+\frac{1}{2}\left(\sum_{k \in\{i, l\}, j \in N \backslash\{i, l\}} \mu(k, j)-2 \sum_{j \in N \backslash\{i, l\}} \mu(j)\right)-\frac{|N|-2}{2}(\mu(i)+\mu(l)) .
$$

By Eq. (8), we have:

$$
\begin{aligned}
\varphi_{\{i, l\}}(\mu, N)-\varphi_{l}(\mu, N)= & \left.\mu(i, l)+\frac{1}{2} \sum_{k \in\{i, l\}, j \in N \backslash\{i, l\}} \mu(k, j)-\frac{1}{2} \sum_{j \in N \backslash l} \mu(l, j)\right)- \\
& \left(\sum_{j \in N \backslash\{i, l\}} \mu(j)-\frac{1}{2} \sum_{j \in N \backslash l} \mu(j)\right)-\frac{|N|-2}{2} \mu(i)-\frac{1}{2} \mu(l)= \\
& \frac{1}{2} \sum_{j \in N \backslash i} \mu(i, j)-\frac{1}{2} \sum_{j \in N \backslash\{i, l\}} \mu(j)+\frac{1}{2} \mu(i)-\frac{|N|-2}{2} \mu(i)-\frac{1}{2} \mu(l)= \\
& \frac{3-|N|}{2} \mu(i)+\frac{1}{2} \sum_{j \in N \backslash i}(\mu(i, j)-\mu(j))=\varphi_{i}(\mu, N) .
\end{aligned}
$$


When $|S| \geq 2$, from Eq. (7) we obtain:

$$
\begin{aligned}
\varphi_{S \cup i}(\mu, N)-\varphi_{S}(\mu, N)= & \sum_{j \in S} \mu(i, j)+\frac{1}{2}\left(\sum_{l \in N \backslash S \cup i} \mu(i, l)-\sum_{j \in S} \mu(i, j)\right)+\frac{|S|}{2} \mu(i)- \\
& \frac{1}{2} \sum_{l \in N \backslash S} \mu(l)-\frac{1}{2} \sum_{j \in S} \mu(j)-\frac{|N|+|S|-3}{2} \mu(i)= \\
& \frac{3-|N|}{2} \mu(i)+\frac{1}{2}\left(\sum_{j \in S} \mu(i, j)+\sum_{l \in N \backslash S \cup i} \mu(i, l)\right)-\frac{1}{2}\left(\sum_{j \in S} \mu(j)+\sum_{l \in N \backslash S} \mu(l)\right)= \\
& \frac{3-|N|}{2} \mu(i)+\frac{1}{2} \sum_{j \in N \backslash i}(\mu(i, j)-\mu(j))=\varphi_{i}(\mu, N) .
\end{aligned}
$$

From Theorem 4, we know that the GABIVIFGC operator is an additive measure, namely, $\varphi_{S}(\mu, N)=\sum_{i \in S} \varphi_{i}(\mu, N)$ for any $S \subseteq N$. Then, it can be expressed by:

$$
\begin{aligned}
& \operatorname{GABIVIFGC}\left(\tilde{\alpha}_{1}, \tilde{\alpha}_{2}, \ldots, \tilde{\alpha}_{n}\right)= \\
& \qquad\left(\left[\prod_{i=1}^{n} a_{i}^{\varphi_{i}(\mu, N)}, \prod_{i=1}^{n} b_{i}^{\varphi} \varphi_{i}(\mu, N)\right],\left[1-\prod_{i=1}^{n}\left(1-c_{i}\right)^{\varphi_{i}(\mu, N)}, 1-\prod_{i=1}^{n}\left(1-d_{i}\right)^{\varphi_{i}(\mu, N)}\right]\right),
\end{aligned}
$$

where $\varphi_{i}(\mu, N)$ is the Banzhaf value of the element $i$ given as Eq. (8).

\section{A new cross entropy measure and the models of obtaining the optimal 2-additive measures}

\subsection{A new cross entropy measure for IVIFSs}

In section 2, we have introduced two aggregation operators for IVIFSs, which are based on the Choquet integral and the generalized Banzhaf index. When the weighting vectors on expert set and criteria set are given, we can develop an approach to multi-criteria group decision making under interval-valued intuitionistic fuzzy environment by using the introduced operators. Otherwise, we first need to get their weighting vectors.

Cross entropy, as a very important method of measuring uncertain information, has been discussed by many researchers. It is used to obtain the weights of criteria (Hung, Yang 2008; Wang, Li 2011; Xia, Xu 2012; Ye 2011; Zhang, Yu 2012).

According to the entropy of a probability distribution introduced by Shannon (1948), Kullback and Leibler (1951) defined the cross entropy measure between two probability distributions $P=\left\{p_{1}, p_{2}, \ldots, p_{n}\right\}$ and $Q=\left\{q_{1}, q_{2}, \ldots, q_{n}\right\}$ as follows:

$$
H(P, Q)=-\sum_{i=1}^{n} p_{i} \log _{2} \frac{p_{i}}{q_{i}} .
$$


Since Eq. (11) is neither symmetric nor satisfied the triangle inequality, it is not a "distance" in the formal sense.

ByEq. (11), for any two fuzzy sets $A=\left\{A\left(x_{i}\right), A\left(x_{2}\right), \ldots, A\left(x_{n}\right)\right\}$ and $B=\left\{B\left(x_{1}\right), B\left(x_{2}\right), \ldots, B\left(x_{n}\right)\right\}$ on the finite set $X=\left\{x_{1}, x_{2}, \ldots, x_{n}\right\}$, Shang and Jiang (1997) proposed a modified fuzzy cross entropy measure:

$$
H_{S J}(A, B)=\sum_{i=1}^{n}\left(A\left(x_{i}\right) \log _{2} \frac{A\left(x_{i}\right)}{\frac{1}{2}\left(A\left(x_{i}\right)+B\left(x_{i}\right)\right)}+\left(1-A\left(x_{i}\right)\right) \log _{2} \frac{1-A\left(x_{i}\right)}{1-\frac{1}{2}\left(B\left(x_{i}\right)+A\left(x_{i}\right)\right)}\right) .
$$

Recently, Ye (2011) gave the cross entropy between two IVIFSs

$$
A=\left\{\left\langle x,\left[\mu_{A}^{-}(x), \mu_{A}^{+}(x)\right],\left[v_{A}^{-}(x), v_{A}^{+}(x)\right]\right\rangle \mid x \in X\right\}
$$

and

as follows:

$$
B=\left\{\left\langle x,\left[\mu_{B}^{-}(x), \mu_{B}^{+}(x)\right],\left[v_{B}^{-}(x), v_{B}^{+}(x)\right]\right\rangle \mid x \in X\right\}
$$

$$
\begin{aligned}
H_{Y}(A, B)= & \sum_{i=1}^{n}\left(\frac{2+\mu_{A}^{+}\left(x_{i}\right)-v_{A}^{+}\left(x_{i}\right)}{4} \log _{2} \frac{2+\mu_{A}^{+}\left(x_{i}\right)-v_{A}^{+}\left(x_{i}\right)}{\frac{1}{2}\left(4+\mu_{A}^{+}\left(x_{i}\right)-v_{A}^{+}\left(x_{i}\right)+\mu_{B}^{+}\left(x_{i}\right)-v_{B}^{+}\left(x_{i}\right)\right)}+\right. \\
& \left.\sum_{i=1}^{n} \frac{2-\mu_{A}^{+}\left(x_{i}\right)+v_{A}^{+}\left(x_{i}\right)}{4} \log _{2} \frac{2-\mu_{A}^{+}\left(x_{i}\right)+v_{A}^{+}\left(x_{i}\right)}{\frac{1}{2}\left(4-\mu_{A}^{+}\left(x_{i}\right)+v_{A}^{+}\left(x_{i}\right)-\mu_{B}^{+}\left(x_{i}\right)+v_{B}^{+}\left(x_{i}\right)\right)}\right)
\end{aligned}
$$

where $\mu_{T}^{+}\left(x_{i}\right)=\mu_{T l}\left(x_{i}\right)+\mu_{T u}\left(x_{i}\right), v_{T}^{+}\left(x_{i}\right)=v_{T l}\left(x_{i}\right)+v_{T u}\left(x_{i}\right)$ for $T=A, B$.

Since $H_{Y}(A, B)$ is not symmetric, Ye (2011) further gave a modified cross entropy measure to a symmetric form between IVIFSs $A$ and $B$ as follows:

$$
H_{Y}^{*}(A, B)=H_{Y}(A, B)+H_{Y}(B, A) .
$$

Eq. (12) can be seen as a "distance" in the formal sense. However, there are still two problems of Eq.(12): on the one hand, for some $x_{i} \in X, H_{Y}(A, B)$ is not a number when $A\left(x_{i}\right)$ and $B\left(x_{i}\right)$ are both crisp values; on the other hand, it only considers the interval-valued membership and non-membership information. Considering the interval-valued hesitation information, we give another cross entropy measure between any two IVIFSs $A$ and $B$ on the finite set $X=\left\{x_{1}, x_{2}, \ldots, x_{n}\right\}$ as follows:

$$
\begin{aligned}
& H(A, B)=\sum_{i=1}^{n} \frac{1}{n}\left(\frac{2+\mu_{A}^{+}\left(x_{i}\right)+P_{\mu_{A}^{+}}\left(x_{i}\right) \pi_{A}^{+}\left(x_{i}\right)-v_{A}^{+}\left(x_{i}\right)-P_{v_{A}^{+}}\left(x_{i}\right) \pi_{A}^{+}\left(x_{i}\right)}{4} \times\right. \\
& \log _{2} \frac{3+\mu_{A}^{+}\left(x_{i}\right)+P_{\mu_{A}^{+}}\left(x_{i}\right) \pi_{A}^{+}\left(x_{i}\right)-v_{A}^{+}\left(x_{i}\right)-P_{v_{A}^{+}}\left(x_{i}\right) \pi_{A}^{+}\left(x_{i}\right)}{\frac{1}{2}\left(6+\mu_{A}^{+}\left(x_{i}\right)+P_{\mu_{A}^{+}}\left(x_{i}\right) \pi_{A}^{+}\left(x_{i}\right)-v_{A}^{+}\left(x_{i}\right)-P_{v_{A}^{+}}\left(x_{i}\right) \pi_{A}^{+}\left(x_{i}\right)+\mu_{B}^{+}\left(x_{i}\right)+P_{\mu_{B}^{+}}\left(x_{i}\right) \pi_{B}^{+}\left(x_{i}\right)-v_{B}^{+}\left(x_{i}\right)-P_{v_{B}^{+}}\left(x_{i}\right) \pi_{B}^{+}\left(x_{i}\right)\right)}+
\end{aligned}
$$




$$
\begin{aligned}
& \frac{2-\mu_{A}^{+}\left(x_{i}\right)-P_{\mu_{A}^{+}}\left(x_{i}\right) \pi_{A}^{+}\left(x_{i}\right)+v_{A}^{+}\left(x_{i}\right)+P_{v_{A}^{+}}\left(x_{i}\right) \pi_{A}^{+}\left(x_{i}\right)}{4} \times \\
& \left.\log _{2} \frac{3-\mu_{A}^{+}\left(x_{i}\right)-P_{\mu_{A}^{+}}\left(x_{i}\right) \pi_{A}^{+}\left(x_{i}\right)+v_{A}^{+}\left(x_{i}\right)+P_{v_{A}^{+}}\left(x_{i}\right) \pi_{A}^{+}\left(x_{i}\right)}{\frac{1}{2}\left(6-\mu_{A}^{+}\left(x_{i}\right)-P_{\mu_{A}^{+}}\left(x_{i}\right) \pi_{A}^{+}\left(x_{i}\right)+v_{A}^{+}\left(x_{i}\right)+P_{v_{A}^{+}}\left(x_{i}\right) \pi_{A}^{+}\left(x_{i}\right)-\mu_{B}^{+}\left(x_{i}\right)-P_{\mu_{B}^{+}}\left(x_{i}\right) \pi_{B}^{+}\left(x_{i}\right)+v_{B}^{+}\left(x_{i}\right)+P_{v_{B}^{+}}\left(x_{i}\right) \pi_{B}^{+}\left(x_{i}\right)\right)}\right),
\end{aligned}
$$

where $\pi_{T}^{+}\left(x_{i}\right)=\pi_{T l}\left(x_{i}\right)+\pi_{T u}\left(x_{i}\right), P_{\mu_{T}^{+}}\left(x_{i}\right)=\frac{\mu_{T}^{+}\left(x_{i}\right)}{2}$ and $P_{v_{T}^{+}}\left(x_{i}\right)=\frac{v_{T}^{+}\left(x_{i}\right)}{2}$ for $T=A, B$.

Remark 2. For any IVIFS $A$, the possibility that the hesitation information tends to be the membership information is $P_{\mu_{A}^{+}}\left(x_{i}\right)=\mu_{A}^{+}\left(x_{i}\right) / 2$, and the possibility that tends to be the non-membership information is $P_{v_{A}^{+}}\left(x_{i}\right)=v_{A}^{+}\left(x_{i}\right) / 2$.

From the relationship between the hesitation information, the membership information and the non-membership information, we know Eq. (13) can be equivalently expressed by:

$$
\begin{aligned}
& H(A, B)=\frac{1}{n} \sum_{i=1}^{n}\left(\frac{2+\left(\mu_{A}^{+}\left(x_{i}\right)-v_{A}^{+}\left(x_{i}\right)\right)\left(1+\pi_{A}^{+}\left(x_{i}\right) / 2\right)}{4} \times\right. \\
& \log _{2} \frac{3+\left(\mu_{A}^{+}\left(x_{i}\right)-v_{A}^{+}\left(x_{i}\right)\right)\left(1+\pi_{A}^{+}\left(x_{i}\right) / 2\right)}{\frac{1}{2}\left(6+\left(\mu_{A}^{+}\left(x_{i}\right)-v_{A}^{+}\left(x_{i}\right)\right)\left(1+\pi_{A}^{+}\left(x_{i}\right) / 2\right)+\left(\mu_{B}^{+}\left(x_{i}\right)-v_{B}^{+}\left(x_{i}\right)\right)\left(1+\pi_{B}^{+}\left(x_{i}\right) / 2\right)\right)}+ \\
& \frac{2+\left(v_{A}^{+}\left(x_{i}\right)-\mu_{A}^{+}\left(x_{i}\right)\right)\left(1+\pi_{A}^{+}\left(x_{i}\right) / 2\right)}{4} \times \\
& \left.\log _{2} \frac{1}{\frac{1}{2}\left(6+\left(v_{A}^{+}\left(x_{i}\right)-\mu_{A}^{+}\left(x_{i}\right)\right)\left(1+x_{A}^{+}\left(x_{i}\right) / 2\right)+\left(v_{B}^{+}\left(x_{i}\right)-\mu_{B}^{+}\left(x_{i}\right)\right)\left(1+\pi_{B}^{+}\left(x_{i}\right) / 2\right)\right)}\right)
\end{aligned}
$$

However, $H(A, B)$ is not symmetric, so it should be modified to a symmetric cross entropy measure as follows:

$$
H^{\star}(A, B)=H(A, B)+H(B, A) .
$$

It is not difficult to find that the cross entropy measure $H^{\star}(A, B)$ between IVIFSs $A$ and $B$ satisfies the following properties:

(i) $H^{\star}(A, B) \geq 0$;

(ii) $H^{\star}(A, B)=0$ if and only if $A=B$;

(iii) $H^{\star}(A, B)=H^{\star}\left(A^{C}, B^{C}\right)$;

(iv) $H^{\star}(A, B)=H^{\star}(B, A)$;

(v) $H^{\star}(A, B) \leq H^{\star}(A, D), H^{\star}(B, D) \leq H^{\star}(A, D)$ for any $D \in \Omega$ with $A \subseteq B \subseteq D$. 


\subsection{The models of obtaining the optimal 2-additive measures}

Now, we consider a multi-criteria group decision making under interval-valued intuitionistic fuzzy environment. Let $A=\left\{a_{1}, a_{2}, \ldots, a_{m}\right\}$ be the set of alternatives, $C=\left\{c_{1}, c_{2}, \ldots, c_{n}\right\}$ be the set of criteria, and $E=\left\{e_{1}, e_{2}, \ldots, e_{q}\right\}$ be the set of experts. Assume that $\left[a_{i j}^{k}, b_{i j}^{k}\right]$ and $\left[c_{i j}^{k}, d_{i j}^{k}\right]$ are the interval degrees of membership and non-membership of the alternative $a_{i}$ satisfies the criterion $c_{j}$ given by the expert $e_{k}$, respectively, where $\left[a_{i j}^{k}, b_{i j}^{k}\right] \subseteq[0,1]$ and $\left[c_{i j}^{k}, d_{i j}^{k}\right] \subseteq[0,1]$ with $b_{i j}^{k}+d_{i j}^{k} \leq 1$. In other words, the evaluation of the alternative $a_{i}$ w.r.t. the criterion $c_{j}$ given by the expert $e_{k}$ is an IVIFV $\tilde{\alpha}_{i j}^{k}=\left(\left[a_{i j}^{k}, b_{i j}^{k}\right],\left[c_{i j}^{k}, d_{i j}^{k}\right]\right)(i=1,2, \ldots, m ; j=1,2, \ldots, n ; k=1,2, \ldots, q)$. By $D^{k}=\left(\tilde{\alpha}_{i j}^{k}\right)_{m \times n}$, we denote the IVIFV matrix given by the expert $e_{k}(k=1,2, \ldots, q)$.

Since the experts' knowledge, skills and experiences are different, it is unreasonable to give an expert the same weight w.r.t. different criteria. Furthermore, the importance of an expert is not only determined by itself, but also influenced by other experts. Namely, it is a relative value. So it is not suitable to give the weights of the experts by using additive measures.

In order to overall reflect the interaction among experts, we shall use their Banzhaf values as their weights. According to the cross entropy principle, the following model is introduced to determine the optimal 2-additive measure on expert set $E$ for the criterion $c_{j}(j=1,2, \ldots, n)$.

$$
\begin{gathered}
\max \sum_{k=1}^{q} \sum_{l=1}^{q} H^{\star}\left(P_{k}^{j}, P_{l}^{j}\right) \varphi_{e_{k}}\left(\mu^{j}, E\right), \\
\sum_{e_{l} \subseteq S \backslash e_{k}}\left(\mu^{j}\left(e_{k}, e_{l}\right)-\mu^{j}\left(e_{l}\right)\right) \geq(|S|-2) \mu^{j}\left(e_{k}\right), \forall S \subseteq E, \forall e_{k} \in S,|S| \geq 2, \\
\sum_{\left\{e_{k}, e_{l}\right\} \subseteq E} \mu^{j}\left(e_{k}, e_{l}\right)-(|E|-2) \sum_{e_{i} \in E} \mu^{j}\left(e_{i}\right)=1, \\
\mu^{j}\left(e_{k}\right) \in W_{e_{k}}^{j}, \mu^{j}\left(e_{k}\right) \geq 0, \quad k=1,2, \ldots, q,
\end{gathered}
$$

where $\varphi_{e_{k}}\left(\mu^{j}, E\right)$ is the Banzhaf value of the expert $e_{k}(k=1,2, \ldots, q), \mu^{j}$ is the 2 -additive measure on expert set $E$ for the criterion $c_{j}, P_{k}^{j}$ is the $j$ th column of the IVIFV matrix $D^{k}$, and $W_{e_{k}}^{j}$ is the range of the expert $e_{k}$ for the criterion $c_{j}(j=1,2, \ldots, n)$.

From Eq. (8), we further have:

$$
\begin{aligned}
& \max \frac{3-q}{2} \sum_{k=1}^{q} \sum_{l=1}^{q} H^{\star}\left(P_{k}^{j}, P_{l}^{j}\right) \mu^{j}\left(e_{k}\right)+\frac{1}{2} \sum_{k=1}^{q} \sum_{l=1}^{q} H^{\star}\left(P_{k}^{j}, P_{l}^{j}\right)\left(\mu^{j}\left(e_{k}, e_{l}\right)-\mu^{j}\left(e_{l}\right)\right), \\
& \text { s.t. }\left\{\begin{array}{l}
\sum_{e_{l} \subseteq S \backslash e_{k}}\left(\mu^{j}\left(e_{k}, e_{l}\right)-\mu^{j}\left(e_{l}\right)\right) \geq(|S|-2) \mu^{j}\left(e_{k}\right), \quad \forall S \subseteq E, \forall e_{k} \in S,|S| \geq 2, \\
\sum_{\left\{e_{k}, e_{l}\right\} \subseteq E} \mu^{j}\left(e_{k}, e_{l}\right)-(|E|-2) \sum_{e_{i} \in E} \mu^{j}\left(e_{i}\right)=1, \\
\mu^{j}\left(e_{k}\right) \in W_{e_{k}}^{j}, \mu^{j}\left(e_{k}\right) \geq 0, \quad k=1,2, \ldots, q .
\end{array}\right.
\end{aligned}
$$

After solving the model (17), we get the optimal 2-additive measure on expert set $E$ for the criterion $c_{i}(j=1,2, \ldots, n)$. By Eq. (8), we get the Banzhaf values of the experts for the criterion $c_{i}(j=1,2, \ldots, n)$, which are used as the experts' weights. 
Similar to the model for the optimal 2-additive measure on expert set, we give the following model for the optimal 2-additive measure on criteria set $C$.

$$
\begin{gathered}
\max \sum_{j=1}^{n} \sum_{l=1}^{n} H^{*}\left(R_{j}, R_{l}\right) \varphi_{c_{j}}(\mu, C), \\
\text { s.t. }\left\{\begin{array}{l}
\sum_{c_{l} \subseteq S \backslash_{c_{j}}}\left(\mu\left(c_{j}, c_{l}\right)-\mu\left(c_{l}\right)\right) \geq(|S|-2) \mu\left(c_{j}\right), \forall S \subseteq C, \forall c_{j} \in S,|S| \geq 2, \\
\sum_{\left\{c_{j}, c_{l}\right\} \subseteq C} \mu\left(c_{j}, c_{l}\right)-(|C|-2) \sum_{c_{j} \in C} \mu\left(c_{j}\right)=1, \\
\mu\left(c_{j}\right) \in W_{c_{j}}, \mu\left(c_{j}\right) \geq 0, \quad j=1,2, \ldots, n,
\end{array}\right.
\end{gathered}
$$

where $\varphi_{c_{j}}(\mu, C)$ is the Banzhaf value of the criterion $c_{j}(j=1,2, \ldots, n), \mu$ is the 2 -additive measure on criteria set $C, R_{j}$ is the $j$ th column of the comprehensive IVIFV matrix $\tilde{R}$, and $W_{c_{j}}$ is the range of the criterion $c_{j}$.

From Eq. (8), we further obtain:

$$
\begin{aligned}
& \max \frac{3-n}{2} \sum_{j=1}^{n} \sum_{l=1}^{n} H^{\star}\left(R_{j}, R_{l}\right) \mu\left(c_{j}\right)+\frac{1}{2} \sum_{j=1}^{n} \sum_{l=1}^{n} H^{\star}\left(R_{j}, R_{l}\right)\left(\mu\left(c_{j}, c_{l}\right)-\mu\left(c_{l}\right)\right), \\
& \text { s.t. }\left\{\begin{array}{l}
\sum_{c_{l} \subseteq S \backslash c_{j}}\left(\mu\left(c_{j}, c_{l}\right)-\mu\left(c_{l}\right)\right) \geq(|S|-2) \mu\left(c_{j}\right), \forall S \subseteq C, \forall c_{j} \in S,|S| \geq 2, \\
\sum_{\left\{c_{j}, c_{l}\right\} \subseteq C} \mu\left(c_{j}, c_{l}\right)-(|C|-2) \sum_{c_{j} \in C} \mu\left(c_{j}\right)=1, \\
\mu\left(c_{j}\right) \in W_{j}, \mu\left(c_{j}\right) \geq 0, \quad j=1,2, \ldots, n .
\end{array}\right.
\end{aligned}
$$

After solving the model (19), we obtain the optimal 2-additive measure on criteria set $C$. By Eq. (8), we get the Banzhaf values of the criteria, which are used as the criteria's weights.

\section{Approaches to pattern recognition and multi-attribute group decision making}

\subsection{An approach to pattern recognition}

Consider a pattern recognition problem. Let $A=\left\{A_{1}, A_{2}, \ldots, A_{m}\right\}$ be the set of the evaluation and selection elements, and $C=\left\{c_{1}, c_{2}, \ldots, c_{n}\right\}$ be the set of features. In practical problems, due to the insufficiency in information availability, it is not easy to identify the exact values of the membership and non-membership. Here, assume that the evaluation of each element $A_{i}$ w.r.t. each feature $c_{j}(j=1,2, \ldots, n)$ is an IVIFV. From analysis above, we develop an approach to some pattern recognition problems under interval-valued intuitionistic fuzzy environment. The decision procedure is described as follows:

Step 1: Suppose that there exist $m$ patterns $A=\left\{A_{1}, A_{2}, \ldots, A_{m}\right\}$ and $n$ features $C=\left\{c_{1}, c_{2}, \ldots, c_{n}\right\}$. The evaluation of each pattern $A_{i}$ w.r.t. the features $C$ is an IVIFS:

$$
A_{i}=\left\{\left\langle\left[b_{i j}^{-}, b_{i j}^{+}\right],\left[d_{i j}^{-}, d_{i j}^{+}\right]\right\rangle \mid j=1,2, \ldots, n\right\} i=1,2, \ldots, m .
$$


Furthermore, assume that there is a sample to be recognized, which is represented by an IVIFS $\varepsilon=\left\{\left\langle\left[e_{j}^{-}, e_{j}^{+}\right],\left[f_{j}^{-}, f_{j}^{+}\right]\right\rangle \mid j=1,2, \ldots, n\right\}$.

Step 2: Use the model (19) to get the optimal 2-additive measure on feature set $C$, and calculate their Banzhaf values by Eq. (8).

Step 3: Calculate the Banzhaf weighted cross entropy measure between $A_{i}$ and $\varepsilon(i=1,2, \ldots, m)$ using $W H^{\star}(A, B)=W H(A, B)+W H(B, A)$, where:

$$
\begin{aligned}
& W H(A, B)=\sum_{i=1}^{n} \varphi_{i}(\mu, C)\left(\frac{2+\left(\mu_{A}^{+}\left(x_{i}\right)-v_{A}^{+}\left(x_{i}\right)\right)\left(1+\pi_{A}^{+}\left(x_{i}\right) / 2\right)}{4} \times\right. \\
& \log _{2} \frac{3+\left(\mu_{A}^{+}\left(x_{i}\right)-v_{A}^{+}\left(x_{i}\right)\right)\left(1+\pi_{A}^{+}\left(x_{i}\right) / 2\right)}{\frac{1}{2}\left(6+\left(\mu_{A}^{+}\left(x_{i}\right)-v_{A}^{+}\left(x_{i}\right)\right)\left(1+\pi_{A}^{+}\left(x_{i}\right) / 2\right)+\left(\mu_{B}^{+}\left(x_{i}\right)-v_{B}^{+}\left(x_{i}\right)\right)\left(1+\pi_{B}^{+}\left(x_{i}\right) / 2\right)\right)}+ \\
& \frac{2+\left(v_{A}^{+}\left(x_{i}\right)-\mu_{A}^{+}\left(x_{i}\right)\right)\left(1+\pi_{A}^{+}\left(x_{i}\right) / 2\right)}{4} \times \\
& \left.\log _{2} \frac{1}{\frac{1}{2}\left(6+\left(v_{A}^{+}\left(x_{i}\right)-\mu_{A}^{+}\left(x_{i}\right)\right)\left(1+\pi_{A}^{+}\left(x_{i}\right) / 2\right)+\left(v_{B}^{+}\left(x_{i}\right)-\mu_{B}^{+}\left(x_{i}\right)\right)\left(1+\pi_{B}^{+}\left(x_{i}\right) / 2\right)\right)}\right)
\end{aligned}
$$

Step 4: Select the smallest one.

Step 5: End.

Example 1. Assume that there are four kinds of minerals $A=\left\{A_{1}, A_{2}, A_{3}, A_{4}\right\}$, and a recognized sample $\varepsilon$, which are represented by the IVIFSs in the feature space $C=\left\{c_{1}, c_{2}, c_{3}\right\}$. Suppose we have the following dates.

$$
\begin{aligned}
& A_{1}=\{\langle[0.2,0.4],[0.4,0.5]\rangle,\langle[0.2,0.3],[0.4,0.6]\rangle,\langle[0.6,0.7],[0.2,0.3]\rangle\}, \\
& A_{2}=\{\langle[0.2,0.3],[0.4,0.6]\rangle,\langle[0.3,0.4],[0.3,0.5]\rangle,\langle[0.3,0.4],[0.4,0.6]\rangle\}, \\
& A_{3}=\{\langle[0.1,0.4],[0.5,0.6]\rangle,\langle[0.2,0.4],[0.4,0.5]\rangle,\langle[0.2,0.3],[0.4,0.6]\rangle\}, \\
& A_{4}=\{\langle[0.2,0.4],[0.4,0.6]\rangle,\langle[0.3,0.4],[0.4,0.6]\rangle,\langle[0.4,0.7],[0.2,0.3]\rangle\}, \\
& \varepsilon=\{\langle[0.2,0.3],[0.4,0.5]\rangle,\langle[0.2,0.3],[0.3,0.5]\rangle,\langle[0.3,0.4],[0.2,0.5]\rangle\} .
\end{aligned}
$$

The importance of features is different, and their range of values are given as $[0.25,0.5]$, $[0.3,0.55]$ and $[0.2,0.35]$. Our purpose is to distinguish which kind of minerals does the recognized sample $\varepsilon$ belong to. In the following, we can utilize the proposed procedure to get the recognized sample $\varepsilon$ belongs to which kind of minerals.

Step 1: By Eq. (15), calculate the cross entropy among $A_{i}^{1}, A_{i}^{2}$ and $A_{i}^{3}(i=1,2,3,4)$, and we get:

$$
H^{\star}\left(A_{i}^{1}, A_{i}^{2}\right)=0.01, H^{\star}\left(A_{i}^{1}, A_{i}^{3}\right)=0.06, H^{\star}\left(A_{i}^{2}, A_{i}^{3}\right)=0.07,
$$

where $A_{i}^{j}$ denotes the $j$ th element of $A_{i}(i=1,2,3,4 ; j=1,2,3)$. 
From the model (19), we get the following linear programming:

$$
\begin{aligned}
& \max -0.035 \mu\left(c_{1}\right)-0.04 \mu\left(c_{2}\right)-0.065 \mu\left(c_{3}\right)+0.01 \mu\left(c_{1}, c_{2}\right)+0.06 \mu\left(c_{1}, c_{3}\right)+0.07 \mu\left(c_{2}, c_{3}\right), \\
& \text { s.t. }\left\{\begin{array}{l}
\mu\left(c_{1}, c_{2}\right)+\mu\left(c_{1}, c_{3}\right)+\mu\left(c_{2}, c_{3}\right)-\mu\left(c_{1}\right)-\mu\left(c_{2}\right)-\mu\left(c_{3}\right)=1, \\
\mu\left(c_{1}\right)-\mu\left(c_{1}, c_{2}\right) \leq 0, \mu\left(c_{2}\right)-\mu\left(c_{1}, c_{2}\right) \leq 0, \\
\mu\left(c_{1}\right)-\mu\left(c_{1}, c_{3}\right) \leq 0, \mu\left(c_{3}\right)-\mu\left(c_{1}, c_{3}\right) \leq 0, \mu\left(c_{2}, c_{3}\right) \leq 0, \mu\left(c_{3}\right)-\mu\left(c_{2}, c_{3}\right) \leq 0, \\
\mu\left(c_{1}\right) \in[0.25,0.5], \\
\mu\left(c_{2}\right) \in[0.3,0.55], \\
\mu\left(c_{3}\right) \in[0.2,0.35] .
\end{array}\right.
\end{aligned}
$$

After solving the above model, we get the optimal 2-additve measure on feature set $C$ as follows:

$$
\mu\left(c_{1}\right)=0.25, \mu\left(c_{2}\right)=0.3, \mu\left(c_{3}\right)=0.2, \mu\left(c_{1}, c_{2}\right)=0.55, \mu\left(c_{1}, c_{3}\right)=0.45, \mu\left(c_{2}, c_{3}\right)=0.75 .
$$

By Eq. (8), we obtain the features' Banzhaf values are:

$$
\varphi_{1}(\mu, C)=0.25, \varphi_{2}(\mu, C)=0.425, \varphi_{3}(\mu, C)=0.325 .
$$

Step 2: Calculate the Banzhaf weighted cross entropy between $A_{i}$ and $\varepsilon(i=1,2,3,4)$, we obtain:

$$
W H^{\star}\left(A_{1}, \varepsilon\right)=0.023, W H^{\star}\left(A_{2}, \varepsilon\right)=0.006, W H^{\star}\left(A_{3}, \varepsilon\right)=0.011, W H^{\star}\left(A_{4}, \varepsilon\right)=0.014 .
$$

Step 3: From step 2, we get $\varepsilon$ belongs to the second kind of minerals.

\subsection{An approach to multi-criteria group decision making}

Based on above analysis, we introduce the following approach to interval-valued intuitionistic fuzzy multi-criteria group decision making. The main decision procedure is described as follows:

Step 1: The expert $e_{k}(k=1,2, \ldots, q)$ evaluates the alternative $a_{i}(i=1,2, \ldots, m)$ w.r.t. each criterion $c_{j}(j=1,2, \ldots, n)$ by an IVIFV $\tilde{\alpha}_{i j}^{k}=\left(\left[a_{i j}^{k}, b_{i j}^{k}\right],\left[c_{i j}^{k}, d_{i j}^{k}\right]\right)(i=1,2, \ldots, m ; j=1,2, \ldots, n$; $k=1,2, \ldots, q)$, and form the IVIFV matrix $D^{k}=\left(\tilde{\alpha}_{i j}^{k}\right)_{m \times n}(k=1,2, \ldots, q)$.

Step 2: If all criteria $c_{j}(j=1,2, \ldots, n)$ are benefit (i.e., the larger, the greater preference), then the criteria values do not need normalization. Otherwise, we normalize the decision matrix $D^{k}=\left(\tilde{\alpha}_{i j}^{k}\right)_{m \times n}$ into $Q^{k}=\left(\tilde{\chi}_{i j}^{k}\right)_{m \times n}(k=1,2, \ldots, q)$, where $\tilde{\chi}_{i j}^{k}=\left\{\begin{array}{l}\tilde{\alpha}_{i j}^{k} \text { for benefit attribute } c_{j} \\ \left(\tilde{\alpha}_{i j}^{k}\right)^{C} \text { for cost attribute } c_{j}\end{array}\right.$ and $\tilde{\chi}_{i j}^{k}=\left(\left[e_{i j}^{k}, f_{i j}^{k}\right],\left[g_{i j}^{k}, h_{i j}^{k}\right]\right)(i=1,2, \ldots, m ; j=1,2, \ldots, n)$ (see Xu, Cai 2010b; Xu, Hu 2010).

Step 3: Use the model (17), calculate the optimal 2-additive measure $\mu^{j}$ on expert set $E$ for each criterion $c_{j}(j=1,2, \ldots, n)$. 
Step 4: By Eq. (8), calculate the Banzhaf values of the experts for each criterion $c_{j}(j=1,2, \ldots, n)$.

Step 5: For each $i=1,2, \ldots, m$ and each $j=1,2, \ldots, n$, use the GABIVIFGC operator:

$$
\begin{aligned}
& \tilde{\gamma}_{i j}=\operatorname{GABIVIFGC}\left(\tilde{\chi}_{i j}^{1}, \tilde{\chi}_{i j}^{2}, \ldots, \tilde{\chi}_{i j}^{q}\right)= \\
& \left(\left[\prod_{k=1}^{q}\left(e_{i j}^{k}\right)^{\varphi_{e_{k}}}{ }^{\left(\mu^{j}, E\right)}, \prod_{k=1}^{q}\left(f_{i j}^{k}\right)^{\varphi_{e_{k}}}\left(\mu^{j}, E\right)\right],\left[1-\prod_{k=1}^{q}\left(1-g_{i j}^{k}\right)^{\varphi_{e_{k}}}{ }^{\left(\mu^{j}, E\right)}, 1-\prod_{k=1}^{q}\left(1-g_{i j}^{k}\right)^{\varphi_{e_{k}}}\left(\mu^{j}, E\right)\right]\right),
\end{aligned}
$$

to get the collective IVIFV $\tilde{\gamma}_{i j}=\left(\left[u_{i j}, v_{i j}\right],\left[x_{i j}, y_{i j}\right]\right)$, and the comprehensive IVIFV matrix $R=\left(\tilde{\gamma}_{i j}\right)_{m \times n}$.

Step 6: Apply the model (19), solve the optimal 2-additive measure $\mu$ on criteria set $C$.

Step 7: By Eq. (8), calculate the Banzhaf values of the criteria.

Step 8: Apply again the GABIVIFGC operator:

$\tilde{\gamma}_{i}=\operatorname{GABIVIFGC}\left(\tilde{\gamma}_{i 1}, \tilde{\gamma}_{i 2}, \ldots, \tilde{\gamma}_{i n}\right)=$

$$
\left(\left[\prod_{j=1}^{n} u_{i j}{ }^{\varphi_{c_{j}}}{ }^{(\mu, C)}, \prod_{j=1}^{n} v_{i j}{ }_{c_{j}}(\mu, C)\right],\left[1-\prod_{j=1}^{n}\left(1-x_{i j}\right)^{\varphi_{c_{j}}(\mu, C)}, 1-\prod_{j=1}^{n}\left(1-y_{i j}\right)^{\varphi_{c_{j}}}(\mu, C)\right]\right),
$$

to get the synthetical value $\tilde{\gamma}_{i}=\left(\left[\alpha_{i l}, \alpha_{i u}\right],\left[\beta_{i l}, \beta_{i u}\right]\right)$ of the alternative $a_{i}(i=1,2, \ldots, m)$.

Step 9: Let

and

$$
\tilde{\gamma}^{+}=\left(\left[\max _{i=1}^{m} \alpha_{i l}, \max _{i=1}^{m} \alpha_{i u}\right],\left[\min _{i=1}^{m} \beta_{i l}, \min _{i=1}^{m} \beta_{i u}\right]\right),
$$

$$
\tilde{\gamma}^{-}=\left(\left[\min _{i=1}^{m} \alpha_{i l}, \min _{i=1}^{m} \alpha_{i u}\right],\left[\max _{i=1}^{m} \beta_{i l}, \max _{i=1}^{m} \beta_{i u}\right]\right) \text {. }
$$

Calculate the cross entropy measures between $H^{\star}\left(\tilde{\gamma}_{i}, \tilde{\gamma}^{+}\right)$and $H^{\star}\left(\tilde{\gamma}_{i}, \tilde{\gamma}^{-}\right)(i=1,2, \ldots, m)$. Step 10: Let $d_{i}=\frac{H^{\star}\left(\tilde{\gamma}_{i}, \tilde{\gamma}^{-}\right)}{H^{\star}\left(\tilde{\gamma}_{i}, \tilde{\gamma}^{-}\right)+H^{\star}\left(\tilde{\gamma}_{i}, \tilde{\gamma}^{+}\right)}(i=1,2, \ldots, m)$. According to $d_{i}(i=1,2, \ldots, m)$, select the biggest one.

Step 11: End.

Example 2. Suppose there is an investment company, which wants to invest a sum of money in the best option (adapted from Ref. Tan 2011b). There is a panel with five possible alternatives to invest the money: $a_{1}$ is a car company; $a_{2}$ is a food company; $a_{3}$ is a computer company; $a_{4}$ is an arms company; $a_{5}$ is a TV company. The investment company must take a decision according to the following four criteria: $c_{1}$ is the risk analysis; $c_{2}$ is the growth analysis; $c_{3}$ is the socio political impact analysis; $c_{4}$ is the environmental impact analysis. The five possible alternatives $a_{i}(i=1,2,3,4,5)$ are to be evaluated using the interval-valued intuitionistic fuzzy information by three decision makers $e_{k}(k=1,2,3)$, as listed in the following matrices: 


$$
\begin{gathered}
D^{1}=\left(\begin{array}{llll}
([0.4,0.5],[0.3,0.4]) & ([0.4,0.6],[0.2,0.4]) & ([0.1,0.3],[0.5,0.6]) & ([0.3,0.4],[0.3,0.5]) \\
([0.6,0.7],[0.2,0.3]) & ([0.6,0.7],[0.2,0.3]) & ([0.4,0.7],[0.1,0.2]) & ([0.5,0.6],[0.1,0.3]) \\
([0.6,0.7],[0.1,0.2]) & ([0.5,0.6],[0.3,0.4]) & ([0.5,0.6],[0.1,0.3]) & ([0.4,0.5],[0.2,0.4]) \\
([0.3,0.4],[0.2,0.3]) & ([0.6,0.7],[0.1,0.3]) & ([0.3,0.4],[0.1,0.2]) & ([0.3,0.7],[0.1,0.2]) \\
([0.7,0.8],[0.1,0.2]) & ([0.3,0.5],[0.1,0.3]) & ([0.5,0.6],[0.2,0.3]) & ([0.3,0.4],[0.5,0.6])
\end{array}\right) \\
D^{2}=\left(\begin{array}{llll}
([0.3,0.4],[0.4,0.5]) & ([0.5,0.6],[0.1,0.3]) & ([0.4,0.5],[0.3,0.4]) & ([0.4,0.6],[0.2,0.4]) \\
([0.3,0.6],[0.3,0.4]) & ([0.4,0.7],[0.1,0.2]) & ([0.5,0.6],[0.2,0.3]) & ([0.6,0.7],[0.2,0.3]) \\
([0.6,0.8],[0.1,0.2]) & ([0.5,0.6],[0.1,0.2]) & ([0.5,0.7],[0.2,0.3]) & ([0.1,0.3],[0.5,0.6]) \\
([0.4,0.5],[0.3,0.5]) & ([0.5,0.8],[0.1,0.2]) & ([0.2,0.5],[0.3,0.4]) & ([0.4,0.7],[0.1,0.2]) \\
([0.6,0.7],[0.2,0.3]) & ([0.6,0.7],[0.1,0.2]) & ([0.5,0.7],[0.2,0.3]) & ([0.6,0.7],[0.1,0.3])
\end{array}\right), \\
D^{3}=\left(\begin{array}{llll}
([0.2,0.5],[0.3,0.4]) & ([0.4,0.5],[0.1,0.2]) & ([0.3,0.6],[0.2,0.3]) & ([0.3,0.7],[0.1,0.3]) \\
([0.2,0.7],[0.2,0.3]) & ([0.3,0.6],[0.2,0.4]) & ([0.4,0.7],[0.1,0.2]) & ([0.5,0.8],[0.1,0.2]) \\
([0.5,0.6],[0.3,0.4]) & ([0.7,0.8],[0.1,0.2]) & ([0.5,0.6],[0.2,0.3]) & ([0.4,0.5],[0.3,0.4]) \\
([0.3,0.6],[0.2,0.4]) & ([0.4,0.6],[0.2,0.3]) & ([0.1,0.4],[0.3,0.6]) & ([0.3,0.7],[0.1,0.2]) \\
([0.6,0.7],[0.1,0.3]) & ([0.5,0.6],[0.3,0.4]) & ([0.5,0.6],[0.2,0.3]) & ([0.5,0.6],[0.2,0.4])
\end{array}\right)
\end{gathered}
$$

Assume that the interval-valued weighting vectors of the experts are respectively given as:

$$
\begin{gathered}
w_{1}=([0.3,0.5],[0.4,0.5],[0.1,0.2],[0.15,0.3]), \\
w_{2}=([0.2,0.3],[0.2,0.3],[0.3,0.4],[0.35,0.5]), \\
w_{3}=([0.25,0.4],[0.2,0.3],[0.25,0.3],[0.3,0.45]),
\end{gathered}
$$

and the interval-valued weighting vector of the criteria is given by $w=([0.3,0.4],[0.15,0.25]$, $[0.2,0.25][0.25,0.3])$. In the following, we can utilize the proposed procedure to get the most desirable alternative(s).

Step 1: Since $c_{2}$ (the growth analysis) is benefit criterion, and $c_{1}$ (the risk analysis), $c_{3}$ (the socio- political impact analysis) and $c_{4}$ (the environmental impact analysis) are cost criteria, we need to normalize the given matrices as listed in the following.

$$
\begin{gathered}
Q^{1}=\left(\begin{array}{llll}
([0.3,0.4],[0.4,0.5]) & ([0.4,0.6],[0.2,0.4]) & ([0.5,0.6],[0.1,0.3]) & ([0.3,0.5],[0.3,0.4]) \\
([0.2,0.3],[0.6,0.7]) & ([0.6,0.7],[0.2,0.3]) & ([0.1,0.2],[0.4,0.7]) & ([0.1,0.3],[0.5,0.6]) \\
([0.1,0.2],[0.6,0.7]) & ([0.5,0.6],[0.3,0.4]) & ([0.1,0.3],[0.5,0.6]) & ([0.2,0.4],[0.4,0.5]) \\
([0.2,0.3],[0.3,0.4]) & ([0.6,0.7],[0.1,0.3]) & ([0.1,0.2],[0.3,0.4]) & ([0.1,0.2],[0.3,0.7]) \\
([0.1,0.2],[0.7,0.8]) & ([0.3,0.5],[0.1,0.3]) & ([0.2,0.3],[0.5,0.6]) & ([0.5,0.6],[0.3,0.4])
\end{array}\right), \\
Q^{2}=\left(\begin{array}{llll}
([0.4,0.5],[0.3,0.4]) & ([0.5,0.6],[0.1,0.3]) & ([0.3,0.4],[0.4,0.5]) & ([0.2,0.4],[0.4,0.6]) \\
([0.3,0.4],[0.3,0.6]) & ([0.4,0.7],[0.1,0.2]) & ([0.2,0.3],[0.5,0.6]) & ([0.2,0.3],[0.6,0.7]) \\
([0.1,0.2],[0.6,0.8]) & ([0.5,0.6],[0.1,0.2]) & ([0.2,0.3],[0.5,0.7]) & ([0.5,0.6],[0.1,0.3]) \\
([0.3,0.5],[0.4,0.5]) & ([0.5,0.8],[0.1,0.2]) & ([0.3,0.4],[0.2,0.5]) & ([0.1,0.2],[0.4,0.7]) \\
([0.2,0.3],[0.6,0.7]) & ([0.6,0.7],[0.1,0.2]) & ([0.2,0.3],[0.5,0.7]) & ([0.1,0.3],[0.6,0.7])
\end{array}\right),
\end{gathered}
$$




$$
Q^{3}=\left(\begin{array}{rrrr}
([0.3,0.4],[0.2,0.5]) & ([0.4,0.5],[0.1,0.2]) & ([0.2,0.3],[0.3,0.6]) & ([0.1,0.3],[0.3,0.7]) \\
([0.2,0.3],[0.2,0.7]) & ([0.3,0.6],[0.2,0.4]) & ([0.1,0.2],[0.4,0.7]) & ([0.1,0.2],[0.5,0.8]) \\
([0.3,0.4],[0.5,0.6]) & ([0.7,0.8],[0.1,0.2]) & ([0.2,0.3],[0.5,0.6]) & ([0.3,0.4],[0.4,0.5]) \\
([0.2,0.4],[0.3,0.6]) & ([0.4,0.6],[0.2,0.3]) & ([0.3,0.6],[0.1,0.4]) & ([0.1,0.2],[0.3,0.7]) \\
([0.1,0.3],[0.6,0.7]) & ([0.5,0.6],[0.3,0.4]) & ([0.2,0.3],[0.5,0.6]) & ([0.2,0.4],[0.5,0.6])
\end{array}\right)
$$

Step 2: By Eq. (15), we get $H^{\star}\left(P_{1}^{1}, P_{2}^{1}\right)=0.06, H^{\star}\left(P_{1}^{1}, P_{3}^{1}\right)=0.062$ and $H^{\star}\left(P_{2}^{1}, P_{3}^{1}\right)=0.065$. From the model (17), we have:

$$
\begin{aligned}
& \max -0.061 \mu^{1}\left(e_{1}\right)-0.0625 \mu^{1}\left(e_{2}\right)-0.0635 \mu^{1}\left(e_{3}\right)+0.06 \mu^{1}\left(e_{1}, e_{2}\right)+0.062 \mu^{1}\left(e_{1}, e_{3}\right)+0.065 \mu^{1}\left(e_{2}, e_{3}\right) \\
& \text { s.t. }\left\{\begin{array}{l}
\mu^{1}\left(e_{1}, e_{2}\right)+\mu^{1}\left(e_{1}, e_{3}\right)+\mu^{1}\left(e_{2}, e_{3}\right)-\mu^{1}\left(e_{1}\right)-\mu^{1}\left(e_{2}\right)-\mu^{1}\left(e_{3}\right)=1, \\
\mu^{1}\left(e_{1}\right)-\mu^{1}\left(e_{1}, e_{2}\right) \leq 0, \mu^{1}\left(e_{2}\right)-\mu^{1}\left(e_{1}, e_{2}\right) \leq 0, \\
\left.\mu^{1}\left(e_{1}\right)-\mu^{1}\left(e_{1}, e_{3}\right) \leq 0, \mu^{1}\left(e_{3}\right)-\mu^{1}\left(e_{1}, e_{3}\right) \leq 0, e_{2}, e_{3}\right) \leq 0, \mu^{1}\left(e_{3}\right)-\mu^{1}\left(e_{2}, e_{3}\right) \leq 0, \\
\mu^{1}\left(e_{1}\right) \in[0.3,0.5], \\
\mu^{1}\left(e_{2}\right) \in[0.2,0.3] \\
\mu^{1}\left(e_{3}\right) \in[0.25,0.4] .
\end{array}\right.
\end{aligned}
$$

After solving the above model, we get the following optimal 2-additve measure $\mu^{1}$ on $E$ for the criterion $c_{1}$ :

$\mu^{1}\left(e_{1}\right)=0.3, \mu^{1}\left(e_{2}\right)=0.2, \mu^{1}\left(e_{3}\right)=0.25, \mu^{1}\left(e_{1}, e_{2}\right)=0.5, \mu^{1}\left(e_{1}, e_{3}\right)=0.55, \mu^{1}\left(e_{2}, e_{3}\right)=0.7$.

Similarly, we have:

$$
\left(\begin{array}{l}
H^{\star}\left(P_{1}^{2}, P_{2}^{2}\right) \\
H^{\star}\left(P_{1}^{2}, P_{3}^{2}\right) \\
H^{\star}\left(P_{2}^{2}, P_{3}^{2}\right)
\end{array}\right)=\left(\begin{array}{l}
0.071 \\
0.097 \\
0.104
\end{array}\right),\left(\begin{array}{l}
H^{\star}\left(P_{1}^{3}, P_{2}^{3}\right) \\
H^{\star}\left(P_{1}^{3}, P_{3}^{3}\right) \\
H^{\star}\left(P_{2}^{3}, P_{3}^{3}\right)
\end{array}\right)=\left(\begin{array}{l}
0.114 \\
0.210 \\
0.031
\end{array}\right),\left(\begin{array}{l}
H^{\star}\left(P_{1}^{4}, P_{2}^{4}\right) \\
H^{\star}\left(P_{1}^{4}, P_{3}^{4}\right) \\
H^{\star}\left(P_{2}^{4}, P_{3}^{4}\right)
\end{array}\right)=\left(\begin{array}{l}
0.271 \\
0.130 \\
0.112
\end{array}\right)
$$

and

$$
\left(\begin{array}{l}
\mu^{2}\left(e_{1}\right) \\
\mu^{2}\left(e_{2}\right) \\
\mu^{2}\left(e_{3}\right) \\
\mu^{2}\left(e_{1}, e_{2}\right) \\
\mu^{2}\left(e_{1}, e_{3}\right) \\
\mu^{2}\left(e_{2}, e_{3}\right)
\end{array}\right)=\left(\begin{array}{l}
0.4 \\
0.2 \\
0.2 \\
0.6 \\
0.6 \\
0.6
\end{array}\right),\left(\begin{array}{l}
\mu^{3}\left(e_{1}\right) \\
\mu^{3}\left(e_{2}\right) \\
\mu^{3}\left(e_{3}\right) \\
\mu^{3}\left(e_{1}, e_{2}\right) \\
\mu^{3}\left(e_{1}, e_{3}\right) \\
\mu^{3}\left(e_{2}, e_{3}\right)
\end{array}\right)=\left(\begin{array}{l}
0.1 \\
0.3 \\
0.25 \\
0.4 \\
0.7 \\
0.55
\end{array}\right),\left(\begin{array}{l}
\mu^{4}\left(e_{1}\right) \\
\mu^{4}\left(e_{2}\right) \\
\mu^{4}\left(e_{3}\right) \\
\mu^{4}\left(e_{1}, e_{2}\right) \\
\mu^{4}\left(e_{1}, e_{3}\right) \\
\mu^{4}\left(e_{2}, e_{3}\right)
\end{array}\right)=\left(\begin{array}{l}
0.15 \\
0.35 \\
0.3 \\
0.7 \\
0.45 \\
0.65
\end{array}\right) .
$$

Step 3: From Step 2 and Eq. (8), we get the Banzhaf values of the experts w.r.t. each criterion as listed in Table 1. 
Table 1. The Banzhaf values of the experts w.r.t. each criterion

\begin{tabular}{lcccc}
\hline & Criterion $c_{1}$ & Criterion $c_{2}$ & Criterion $c_{3}$ & Criterion $c_{4}$ \\
\hline The Banzhaf value $\varphi_{e_{1}}$ of the expert $e_{1}$ & 0.3 & 0.4 & 0.275 & 0.25 \\
\hline The Banzhaf value $\varphi_{e_{2}}$ of the expert $e_{2}$ & 0.325 & 0.3 & 0.3 & 0.45 \\
\hline The Banzhaf value $\varphi_{e_{3}}$ of the expert $e_{3}$ & 0.375 & 0.3 & 0.425 & 0.3 \\
\hline
\end{tabular}

Step 4: Use the GABIVIFGC operator, we get the following comprehensive IVIFV matrix

$$
R=\left(\begin{array}{l}
([0.33,0.43],[0.30,0.47])([0.43,0.57],[0.14,0.31])([0.29,0.40],[0.28,0.50])([0.18,0.39],[0.35,0.59]) \\
([0.32,0.67],[0.23,0.33])([0.43,0.67],[0.17,0.30])([0.12,0.23],[0.43,0.67])([0.14,0.27],[0.55,0.71]) \\
([0.56,0.69],[0.18,0.28])([0.55,0.65],[0.19,0.29])([0.17,0.30],[0.50,0.63])([0.34,0.48],[0.28,0.42]) \\
([0.33,0.50],[0.23,0.41])([0.50,0.70],[0.13,0.27])([0.22,0.39],[0.19,0.43])([0.10,0.20],[0.34,0.70]) \\
([0.63,0.73],[0.13,0.27])([0.43,0.58],[0.17,0.30])([0.20,0.30],[0.50,0.63])([0.18,0.39],[0.51,0.61])
\end{array}\right) .
$$

Step 5: From the comprehensive IVIFV matrix $R=\left(\tilde{\gamma}_{i j}\right)_{m \times n}$ and Eq. (15), we have:

$$
\begin{aligned}
& H^{\star}\left(R_{1}, R_{2}\right)=0.085, H^{\star}\left(R_{1}, R_{3}\right)=0.557, H^{\star}\left(R_{1}, R_{4}\right)=0.496, \\
& H^{\star}\left(R_{2}, R_{3}\right)=0.607, H^{\star}\left(R_{2}, R_{4}\right)=0.680, H^{\star}\left(R_{3}, R_{4}\right)=0.130 .
\end{aligned}
$$

By the model (19), we have:

$$
\begin{gathered}
\quad \max -1.138 \mu\left(c_{1}\right)-1.372 \mu\left(c_{2}\right)-1.294 \mu\left(c_{3}\right)-1.306 \mu\left(c_{4}\right)+0.085 \mu\left(c_{1}, c_{2}\right)+ \\
0.557 \mu\left(c_{1}, c_{3}\right)+0.496 \mu\left(c_{1}, c_{4}\right)+0.607 \mu\left(c_{2}, c_{3}\right)+0.68 \mu\left(c_{2}, c_{4}\right)+0.13 \mu\left(c_{3}, c_{4}\right) \\
\qquad \begin{array}{l}
\mu\left(c_{1}\right)+\mu\left(c_{2}\right)-\mu\left(c_{1}, c_{2}\right) \leq 0, \mu\left(c_{1}\right)+\mu\left(c_{3}\right)-\mu\left(c_{1}, c_{3}\right) \leq 0, \\
\mu\left(c_{1}\right)+\mu\left(c_{4}\right)-\mu\left(c_{1}, c_{4}\right) \leq 0, \mu\left(e_{2}\right)+\mu\left(e_{3}\right)-\mu\left(e_{2}, e_{3}\right) \leq 0, \\
\mu\left(c_{2}\right)+\mu\left(c_{4}\right)-\mu\left(c_{2}, c_{4}\right) \leq 0, \mu\left(e_{3}\right)+\mu\left(e_{4}\right)-\mu\left(e_{3}, e_{4}\right) \leq 0, \\
\mu\left(c_{1}\right)+\mu\left(c_{2}\right)+\mu\left(c_{3}\right)-\mu\left(c_{1}, c_{2}\right)-\mu\left(c_{1}, c_{3}\right) \leq 0, \\
\mu\left(c_{1}\right)+\mu\left(c_{2}\right)+\mu\left(c_{3}\right)-\mu\left(c_{1}, c_{2}\right)-\mu\left(c_{2}, c_{3}\right) \leq 0, \\
\mu\left(c_{1}\right)+\mu\left(c_{2}\right)+\mu\left(c_{3}\right)-\mu\left(c_{1}, c_{3}\right)-\mu\left(c_{2}, c_{3}\right) \leq 0, \\
\mu\left(c_{1}\right)+\mu\left(c_{2}\right)+\mu\left(c_{4}\right)-\mu\left(c_{1}, c_{2}\right)-\mu\left(c_{1}, c_{4}\right) \leq 0, \\
\mu\left(c_{1}\right)+\mu\left(c_{2}\right)+\mu\left(c_{4}\right)-\mu\left(c_{1}, c_{2}\right)-\mu\left(c_{2}, c_{4}\right) \leq 0, \\
\mu\left(c_{1}\right)+\mu\left(c_{2}\right)+\mu\left(c_{4}\right)-\mu\left(c_{1}, c_{4}\right)-\mu\left(c_{2}, c_{4}\right) \leq 0, \\
\mu\left(c_{1}\right)+\mu\left(c_{3}\right)+\mu\left(c_{4}\right)-\mu\left(c_{1}, c_{3}\right)-\mu\left(c_{1}, c_{4}\right) \leq 0, \\
\mu\left(c_{1}\right)+\mu\left(c_{3}\right)+\mu\left(c_{4}\right)-\mu\left(c_{1}, c_{3}\right)-\mu\left(c_{3}, c_{4}\right) \leq 0, \\
\mu\left(c_{1}\right)+\mu\left(c_{3}\right)+\mu\left(c_{4}\right)-\mu\left(c_{2}, c_{4}\right)-\mu\left(c_{3}, c_{4}\right) \leq 0, \\
\mu\left(c_{2}\right)+\mu\left(c_{3}\right)+\mu\left(c_{4}\right)-\mu\left(c_{2}, c_{3}\right)-\mu\left(c_{2}, c_{4}\right) \leq 0, \\
\mu\left(c_{2}\right)+\mu\left(c_{3}\right)+\mu\left(c_{4}\right)-\mu\left(c_{2}, c_{3}\right)-\mu\left(c_{3}, c_{4}\right) \leq 0, \\
\mu\left(c_{2}\right)+\mu\left(c_{3}\right)+\mu\left(c_{4}\right)-\mu\left(c_{2}, c_{4}\right)-\mu\left(c_{3}, c_{4}\right) \leq 0, \\
\mu\left(c_{1}, c_{2}\right)+\mu\left(c_{1}, c_{3}\right)+\mu\left(c_{1}, c_{4}\right)+\mu\left(c_{2}, c_{3}\right)+\mu\left(c_{2}, c_{4}\right) \\
+\mu\left(c_{3}, c_{4}\right)-2\left(\mu\left(c_{1}\right)+\mu\left(c_{2}\right)+\mu\left(c_{3}\right)+\mu\left(c_{4}\right)\right)=1, \\
\mu\left(c_{1}\right) \in[0.3,0.4], \mu\left(c_{2}\right) \in[0.15,0.25], \mu\left(c_{3}\right) \in[0.2,0.25], \mu\left(c_{4}\right) \in[0.25,0.3] .
\end{array}
\end{gathered}
$$


After solving e the above model, we get the following optimal 2-additve measure $\mu$ on criteria set $C$ :

$$
\begin{aligned}
& \mu\left(c_{1}\right)=0.3, \mu\left(c_{2}\right)=0.15, \mu\left(c_{3}\right)=0.2, \mu\left(c_{4}\right)=0.25, \mu\left(c_{1}, c_{2}\right)=0.45, \mu\left(c_{1}, c_{3}\right)=0.5, \mu\left(c_{1}, c_{4}\right)=0.55, \\
& \mu\left(c_{2}, c_{3}\right)=0.35, \mu\left(c_{2}, c_{4}\right)=0.5, \mu\left(c_{3}, c_{4}\right)=0.45 .
\end{aligned}
$$

Step 6: From Eq. (8), we obtain the criteria Banzhaf values:

$$
\varphi_{1}(\mu, C)=0.3, \varphi_{2}(\mu, C)=0.2, \varphi_{3}(\mu, C)=0.2, \varphi_{4}(\mu, C)=03 .
$$

Step 7: Use again the GABIVIFGC operator, the synthetical IVIFVs of the criteria are obtained by:

$$
\left(\begin{array}{c}
\tilde{\gamma}_{1} \\
\tilde{\gamma}_{2} \\
\tilde{\gamma}_{3} \\
\tilde{\gamma}_{4} \\
\tilde{\gamma}_{5}
\end{array}\right)=\left(\begin{array}{c}
([0.28,0.44],[0.28,0.49]) \\
([0.22,0.41],[0.37,0.54]) \\
([0.38,0.52],[0.29,0.41]) \\
([0.23,0.39],[0.24,0.50]) \\
([0.32,0.48],[0.35,0.48])
\end{array}\right) .
$$

Step 8: By $\tilde{\gamma}_{i}(i=1,2,3,4,5)$, we get $\tilde{\gamma}^{+}=([0.38,0.52],[0.24,0.41])$ and $\tilde{\gamma}^{-}=([0.22,0.39]$, $[0.37,0.54])$. Calculate the cross entropy between $\tilde{\gamma}_{i}(i=1,2,3,4,5)$ and $\tilde{\gamma}^{+}$,

$$
\left(\begin{array}{c}
H^{\star}\left(\tilde{\gamma}_{1}, \tilde{\gamma}^{+}\right) \\
H^{\star}\left(\tilde{\gamma}_{2}, \tilde{\gamma}^{+}\right) \\
H^{\star}\left(\tilde{\gamma}_{3}, \tilde{\gamma}^{+}\right) \\
H^{\star}\left(\tilde{\gamma}_{4}, \tilde{\gamma}^{+}\right) \\
H^{\star}\left(\tilde{\gamma}_{5}, \tilde{\gamma}^{+}\right)
\end{array}\right)=\left(\begin{array}{l}
0.044 \\
0.136 \\
0.001 \\
0.069 \\
0.038
\end{array}\right),
$$

and calculate the cross entropy between $\tilde{\gamma}_{i}(i=1,2,3,4,5)$ and $\tilde{\gamma}^{-}$,

$$
\left(\begin{array}{c}
H^{\star}\left(\tilde{\gamma}_{1}, \tilde{\gamma}^{-}\right) \\
H^{\star}\left(\tilde{\gamma}_{2}, \tilde{\gamma}^{-}\right) \\
H^{\star}\left(\tilde{\gamma}_{3}, \tilde{\gamma}^{-}\right) \\
H^{\star}\left(\tilde{\gamma}_{4}, \tilde{\gamma}^{-}\right) \\
H^{\star}\left(\tilde{\gamma}_{5}, \tilde{\gamma}^{-}\right)
\end{array}\right)=\left(\begin{array}{l}
0.031 \\
0.000 \\
0.120 \\
0.015 \\
0.037
\end{array}\right) .
$$

Step 9: From $H^{\star}\left(\tilde{\gamma}_{i}, \tilde{\gamma}^{+}\right)$and $H^{\star}\left(\tilde{\gamma}_{i}, \tilde{\gamma}^{-}\right)(i=1,2,3,4,5)$, we obtain:

$$
d_{1}=0.41, d_{2}=0, d_{3}=0.99, d_{4}=0.18, d_{5}=0.49 \text {. }
$$

Thus, $d_{3}>d_{5}>d_{1}>d_{4}>d_{2}$. Namely, $a_{3}$ (computer company) is the best choice.

In Step 7, if we adopt the order relationship between IVIFVs given by Xu (2007c), then we have $\tilde{\gamma}_{3}>\tilde{\gamma}_{5}>\tilde{\gamma}_{1}>\tilde{\gamma}_{4}>\tilde{\gamma}_{2}$, which is the same as the ranking results above. 
In Step 8, if we let $\tilde{\gamma}^{+}=([1,1],[0,0])$ and $\tilde{\gamma}^{-}=([0,0],[1,1])$, then we get the following cross entropy measures:

$$
\left(\begin{array}{l}
H^{\prime \star}\left(\tilde{\gamma}_{1}, \tilde{\gamma}^{+}\right) \\
H^{\prime \star}\left(\tilde{\gamma}_{2}, \tilde{\gamma}^{+}\right) \\
H^{\prime \star}\left(\tilde{\gamma}_{3}, \tilde{\gamma}^{+}\right) \\
H^{\prime \star}\left(\tilde{\gamma}_{4}, \tilde{\gamma}^{+}\right) \\
H^{\prime \star}\left(\tilde{\gamma}_{5}, \tilde{\gamma}^{+}\right)
\end{array}\right)=\left(\begin{array}{l}
0.095 \\
0.112 \\
0.065 \\
0.108 \\
0.044
\end{array}\right),\left(\begin{array}{l}
H^{\prime \star}\left(\tilde{\gamma}_{1}, \tilde{\gamma}^{-}\right) \\
H^{\prime \star}\left(\tilde{\gamma}_{2}, \tilde{\gamma}^{-}\right) \\
H^{\prime \star}\left(\tilde{\gamma}_{3}, \tilde{\gamma}^{-}\right) \\
H^{\prime \star}\left(\tilde{\gamma}_{4}, \tilde{\gamma}^{-}\right) \\
H^{\prime \star}\left(\tilde{\gamma}_{5}, \tilde{\gamma}^{-}\right)
\end{array}\right)=\left(\begin{array}{c}
0.075 \\
0.061 \\
0.107 \\
0.063 \\
0.118
\end{array}\right) .
$$

From $H^{\prime \star}\left(\tilde{\gamma}_{i}, \tilde{\gamma}^{+}\right)$and $H^{\prime \star}\left(\tilde{\gamma}_{i}, \tilde{\gamma}^{-}\right)(i=1,2,3,4,5)$, we have:

$$
d_{1}=0.44, d_{2}=0.35, d_{3}=0.62, d_{4}=0.37, d_{5}=0.73 \text {. }
$$

Thus, $d_{5}>d_{3}>d_{1}>d_{4}>d_{2}$. Namely, $a_{5}$ (TV company) is the best choice, which is different to the ranking results obtained above.

From this example, we can find that some disadvantages for using $([1,1],[0,0])$ and $([0,0],[1,1])$ as the positive and negative ideal points, respectively, actually exist.

In this example, if the IVIFCA operator (Xu 2010) is applied to calculate the comprehensive IVIFV matrix, then it has:

$$
R_{1}=\left(\begin{array}{l}
([0.32,0.42],[0.27,0.48])([0.42,0.56],[0.13,0.29])([0.27,0.37],[0.29,0.53])([0.19,0.39],[0.35,0.59]) \\
([0.22,0.32],[0.30,0.68])([0.46,0.66],[0.20,0.34])([0.13,0.23],[0.43,0.67])([0.16,0.27],[0.55,0.71]) \\
([0.15,0.26],[0.57,0.72])([0.55,0.65],[0.16,0.26])([0.16,0.30],[0.50,0.63])([0.35,0.48],[0.25,0.42]) \\
([0.22,0.39],[0.32,0.51])([0.51,0.69],[0.13,0.28])([0.22,0.38],[0.20,0.43])([0.10,0.20],[0.35,0.70]) \\
([0.12,0.27],[0.63,0.73])([0.45,0.59],[0.16,0.31])([0.20,0.30],[0.50,0.63])([0.20,0.39],[0.51,0.61])
\end{array}\right) .
$$

Furthermore, if the IVIFCA operator (Xu 2010) is applied to calculate the comprehensive IVIFV $\tilde{z}_{i}$ of the alternative $a_{i}(i=1,2,3,4,5)$, then it has:

$$
\left(\begin{array}{l}
\tilde{z}_{1} \\
\tilde{z}_{2} \\
\tilde{z}_{3} \\
\tilde{z}_{4} \\
\tilde{z}_{5}
\end{array}\right)=\left(\begin{array}{c}
([0.30,0.43],[0.26,0.48]) \\
([0.23,0.36],[0.35,0.62]) \\
([0.28,0.40],[0.30,0.47]) \\
([0.25,0.41],[0.26,0.49]) \\
([0.21,0.37],[0.39,0.60])
\end{array}\right)
$$

According to the comprehensive IVIFVs $\tilde{z}_{i}(i=1,2,3,4,5)$, we get the following cross entropy:

$$
\left(\begin{array}{c}
H^{\star}\left(\tilde{z}_{1}, \tilde{z}^{+}\right) \\
H^{\star}\left(\tilde{z}_{2}, \tilde{z}^{+}\right) \\
H^{\star}\left(\tilde{z}_{3}, \tilde{z}^{+}\right) \\
H^{\star}\left(\tilde{z}_{4}, \tilde{z}^{+}\right) \\
H^{\star}\left(\tilde{z}_{5}, \tilde{z}^{+}\right)
\end{array}\right)=\left(\begin{array}{c}
0.0000 \\
0.0171 \\
0.0008 \\
0.0011 \\
0.0194
\end{array}\right),\left(\begin{array}{l}
H^{\star}\left(\tilde{z}_{1}, \tilde{z}^{-}\right) \\
H^{\star}\left(\tilde{z}_{2}, \tilde{z}^{-}\right) \\
H^{\star}\left(\tilde{z}_{3}, \tilde{z}^{-}\right) \\
H^{\star}\left(\tilde{z}_{4}, \tilde{z}^{-}\right) \\
H^{\star}\left(\tilde{z}_{5}, \tilde{z}^{-}\right)
\end{array}\right)=\left(\begin{array}{c}
0.0235 \\
0.0007 \\
0.0179 \\
0.0178 \\
0.0005
\end{array}\right),
$$

where $\tilde{z}^{+}=([0.30,0.43],[0.26,0.47])$ and $\tilde{z}^{-}=([0.21,0.36],[0.39,0.62])$. 
From $H^{\star}\left(\tilde{z}_{i}, \tilde{z}^{+}\right)$and $H^{\star}\left(\tilde{z}_{i}, \tilde{z}^{-}\right)(i=1,2,3,4,5)$, we have:

$$
d_{1}=1, d_{2}=0.04, d_{3}=0.96, d_{4}=0.94, d_{5}=0.02
$$

Thus, $d_{1}>d_{3}>d_{4}>d_{2}>d_{5}$. Namely, $a_{1}$ (car company) is the best choice.

In this example, if the IVIFGA operator (Xu 2010; Tan 2011b) is applied to calculate the comprehensive matrix, then it has:

$$
R_{2}=\left(\begin{array}{l}
([0.32,0.42],[0.29,0.48])([0.42,0.56],[0.14,0.31])([0.25,0.35],[0.31,0.55])([0.17,0.38],[0.36,0.61]) \\
([0.22,0.32],[0.37,0.68])([0.42,0.66],[0.20,0.34])([0.12,0.23],[0.43,0.67])([0.15,0.27],[0.56,0.72]) \\
([0.13,0.24],[0.58,0.73])([0.53,0.64],[0.19,0.29])([0.15,0.30],[0.50,0.63])([0.31,0.46],[0.31,0.44]) \\
([0.22,0.38],[0.32,0.53])([0.49,0.68],[0.14,0.28])([0.18,0.32],[0.22,0.43])([0.10,0.20],[0.36,0.70]) \\
([0.11,0.27],[0.63,0.73])([0.42,0.58],[0.19,0.32])([0.20,0.30],[0.50,0.63])([0.16,0.36],[0.53,0.64])
\end{array}\right) .
$$

Furthermore, if the IVIFGA operator (Xu 2010; Tan 2011b) is applied to calculate the comprehensive IVIFV $\tilde{z}_{i}$ of the alternative $a_{i}(i=1,2,3,4,5)$, then it has:

$$
\left(\begin{array}{l}
\tilde{z}_{1} \\
\tilde{z}_{2} \\
\tilde{z}_{3} \\
\tilde{z}_{4} \\
\tilde{z}_{5}
\end{array}\right)=\left(\begin{array}{c}
([0.25,0.41],[0.30,0.52]) \\
([0.19,0.31],[0.44,0.66]) \\
([0.21,0.37],[0.43,0.57]) \\
([0.18,0.32],[0.29,0.55]) \\
([0.17,0.34],[0.52,0.63])
\end{array}\right)
$$

According to the comprehensive IVIFVs $\tilde{z}_{i}(i=1,2,3,4,5)$, we get the following cross entropy:

$$
\left(\begin{array}{c}
H^{\star}\left(\tilde{z}_{1}, \tilde{z}^{+}\right) \\
H^{\star}\left(\tilde{z}_{2}, \tilde{z}^{+}\right) \\
H^{\star}\left(\tilde{z}_{3}, \tilde{z}^{+}\right) \\
H^{\star}\left(\tilde{z}_{4}, \tilde{z}^{+}\right) \\
H^{\star}\left(\tilde{z}_{5}, \tilde{z}^{+}\right)
\end{array}\right)=\left(\begin{array}{l}
0.0000 \\
0.0215 \\
0.0075 \\
0.0068 \\
0.0242
\end{array}\right),\left(\begin{array}{l}
H^{\star}\left(\tilde{z}_{1}, \tilde{z}^{-}\right) \\
H^{\star}\left(\tilde{z}_{2}, \tilde{z}^{-}\right) \\
H^{\star}\left(\tilde{z}_{3}, \tilde{z}^{-}\right) \\
H^{\star}\left(\tilde{z}_{4}, \tilde{z}^{-}\right) \\
H^{\star}\left(\tilde{z}_{5}, \tilde{z}^{-}\right)
\end{array}\right)=\left(\begin{array}{l}
0.0387 \\
0.0033 \\
0.0110 \\
0.0233 \\
0.0002
\end{array}\right),
$$

where $\tilde{z}^{+}=([0.25,0.41],[0.29,0.52])$ and $\tilde{z}^{-}=([0.17,0.31],[0.52,0.66])$.

From $H^{\star}\left(\tilde{z}_{i}, \tilde{z}^{+}\right)$and $H^{\star}\left(\tilde{z}_{i}, \tilde{z}^{-}\right)(i=1,2,3,4,5)$, we have:

$$
d_{1}=1, d_{2}=0.13, d_{3}=0.59, d_{4}=0.77, d_{5}=0.01
$$

Thus, $d_{1}>d_{4}>d_{3}>d_{2}>d_{5}$. Namely, $a_{1}$ (car company) is the best choice. With respect to the different aggregation operators, ranking results are obtained as shown in Table 2. 
Table 2. Ranking results with respect to the different aggregation operators

\begin{tabular}{ll}
\hline \multicolumn{1}{c}{ Operator } & Ranking order \\
\hline The GABIVIFGC operator & $d_{3}>d_{5}>d_{1}>d_{4}>d_{2}$ \\
\hline The IVIFCA operator & $d_{1}>d_{3}>d_{4}>d_{2}>d_{5}$ \\
\hline The IVIFGA operator & $d_{1}>d_{4}>d_{3}>d_{2}>d_{5}$ \\
\hline
\end{tabular}

The ranking results show that different optimal alternatives may be yielded by using different aggregation operators, and thus, the decision makers can properly select the aggregation operators according to the underlying interest and demands to each individual problem.

\section{Conclusions}

When we deal with the real decision making problems, it is unreasonable to use the additive measures because the independence among elements in a set usually violates. Based on the Choquet integral and the generalized Banzhaf index, we put forward the GBIVIFGC operator, which not only globally considers the importance of elements or their ordered positions, but also overall reflects the correlations among them or their ordered positions. In order to simplify the complexity of solving a fuzzy measure, we further introduce the GBIVIFGC operator w.r.t. 2- additive measure. Moreover, we give a new cross entropy measure for IVIFSs. When the information about weights of experts and criteria is partly known, the models of getting the optimal 2-additive measures on them are presented, respectively. Then, we develop an approach to pattern recognition and multi-criteria group decision making under interval-valued intuitionistic fuzzy environment. And one thing which is worth pointing out is that when the features, the criteria and the experts are respectively independent, we can get the corresponding methods based on additive measures.

\section{Acknowledgments}

This work was supported by the State Key Program of National Natural Science of China (No. 71431006), the Funds for Creative Research Groups of China (No. 71221061), the Projects of Major International Cooperation NSFC (No. 71210003), the National Natural Science Foundation of China (Nos. 71201089, 71271217, 71201110 and 71271029), the National Science Foundation for Post-doctoral Scientists of China (2014M560655), and the Program for New Century Excellent Talents in University of China (No. NCET-12-0541).

\section{References}

Atanassov, K. T. 1986. Intuitionistic fuzzy sets, Fuzzy Sets and Systems 20(1): 87-96. http://dx.doi.org/10.1016/S0165-0114(86)80034-3

Atanassov, K. T.; Gargov, G. 1989. Interval valued intuitionistic fuzzy sets, Fuzzy Sets and Systems 31(3): 343-349. http://dx.doi.org/10.1016/0165-0114(89)90205-4 
Atanassov, K. T. 1994. Operators over interval-valued intuitionistic fuzzy sets, Fuzzy Sets and Systems 64(2): 159-174. http://dx.doi.org/10.1016/0165-0114(94)90331-X

Atanassov, K. T. 1999. Intuitionistic fuzzy sets: theory and applications. Heidelberg: Physica-Verlag. http://dx.doi.org/10.1007/978-3-7908-1870-3

Banzhaf, J. 1965. Weighted voting does not work: a mathematical analysis, Rutgers Law Review 19:317-343.

Bustince, H.; Herrera, F.; Montero, J. 2007. Fuzzy sets and their extensions: representation, aggregation, and models. Heidelberg: Springer-Verlag.

Chen, T. Y.; Li, C. H. 2010. Determining objective weights with intuitionistic fuzzy entropy measures: a comparative analysis, Information Sciences 180(21): 4207-4222. http://dx.doi.org/10.1016/j.ins.2010.07.009

Chen, T. Y.; Hsiao, P. W.; Yen, Y. L. 2011. A multicriteria group decision-making approach based on interval-valued intuitionistic fuzzy sets: a comparative perspective, Expert Systems with Applications 38(6): 7647-7658. http://dx.doi.org/10.1016/j.eswa.2010.12.096

Choquet, G. 1953. Theory of capacities, Annales de l'institut Fourier 5: 131-295.

De, S. K.; Biswas, R.; Roy, A. R. 2001. An application of intuitionistic fuzzy sets in medical diagnosis, Fuzzy Sets and Systems 117(3): 209-213. http://dx.doi.org/10.1016/S0165-0114(98)00235-8

Dubois, D.; Prade, H. 1988. Possibility theory: an approach to computerized processing of uncertainty. New York: Plenum Press. http://dx.doi.org/10.1007/978-1-4684-5287-7

Grabisch, M. 1995. Fuzzy integral in multicriteria decision making, Fuzzy Sets and Systems 69(3): 279-298. http://dx.doi.org/10.1016/0165-0114(94)00174-6

Grabisch, M. 1996. The application of fuzzy integrals in multicriteria decision making, European Journal of Operational Research 89(3): 445-456. http://dx.doi.org/10.1016/0377-2217(95)00176-X

Grabisch, M. 1997. $k$-order additive discrete fuzzy measures and their representation, Fuzzy Sets and Systems 92(2): 167-189. http://dx.doi.org/10.1016/S0165-0114(97)00168-1

Grabisch, M.; Roubens, M. 1999. An axiomatic approach to the concept of interaction among players in cooperative games, International Journal of Game Theory 28(4): 547-565. http://dx.doi.org/10.1007/s001820050125

Grabisch, M.; Murofushi, T.; Sugeno, M. 2000. Fuzzy measure and integrals. New York: Physica-Verlag.

Grabisch, M.; Labreuche, C. 2008. A decade of application of the Choquet and Sugeno integrals in multicriteria decision aid, Annals of Operations Research 6(1): 1-44. http://dx.doi.org/10.1007/s10479-009-0655-8

Hung, W. L.; Yang, M. S. 2008. On the J-divergence of intuitionistic fuzzy sets with its application to pattern recognition, Information Sciences 178(6): 1641-1650. http://dx.doi.org/10.1080/03081070600574353

Jiang, Y. C.; Tang, Y.; Wang, J.; Tang, S. 2009. Reasoning within intuitionistic fuzzy rough description logics, Information Sciences 179(14): 2362-2378. http://dx.doi.org/10.1016/j.ins.2009.03.001

Kojadinovic, I. 2003. Modeling interaction phenomena using fuzzy measures: on the notions of interaction and independence, Fuzzy Sets and Systems 135(3): 317-340. http://dx.doi.org/10.1016/S0165-0114(02)00129-X

Kojadinovic, I. 2005. An axiomatic approach to the measurement of the amount of interaction among criteria or players, Fuzzy Sets and Systems 152(3): 417-435. http://dx.doi.org/10.1016/j.fss.2004.11.006

Kullback, S.; Leibler, R. A. 1951. On information and sufficiency, Annals of Mathematical Statistics 4: 99-111.

Labreuche, C.; Grabisch, M. 2003. The Choquet integral for the aggregation of interval scales in multicriteria decision making, Fuzzy Sets and Systems 137(1): 11-26.

http://dx.doi.org/10.1016/S0165-0114(02)00429-3

Li, D. F. 2010. TOPSIS-based nonlinear-programming methodology for multiattribute decision making with interval-valued intuitionistic, IEEE Transactions on Fuzzy Systems 18(2): 299-311.

http://dx.doi.org/10.1109/TFUZZ.2010.2041009 
Li, D. F. 2011a. Extension principles for interval-valued intuitionistic fuzzy sets and algebraic operations, Fuzzy Optimization and Decision Making 10(1): 45-58. http://dx.doi.org/10.1007/s10700-010-9095-9

Li, D. F. 2011b. Closeness coefficient based nonlinear programming method for interval-valued intuitionistic fuzzy multiattibute decision making with incomplete preference information, Applied Soft Computing 11(4): 3402-3418. http://dx.doi.org/10.1016/j.asoc.2011.01.011

Marichal, J. L. 2000. The influence of variables on pseudo-Boolean functions with applications to game theory and multicriteria decision making, Discrete Applied Mathematics 107(1-3): 139-164. http://dx.doi.org/10.1016/S0166-218X(00)00264-X

Miranda, P.; Grabisch, M.; Gil, P. 2002. p-Symmetric fuzzy measures, International Journal of Uncertainty, Fuzziness, and Knowledge-Based Systems 10(Suppl.): 105-123. http://dx.doi.org/10.1142/S0218488502001867

Nayagam, V. L. G.; Sivaraman, G. 2011. Ranking of interval-valued intuitionistic fuzzy sets, Applied Soft Computing 11(4): 3368-3372. http://dx.doi.org/10.1016/j.asoc.2011.01.008

Park, J. H.; Park, Y.; Young, C. K.; Xue, T. 2011. Extension of the TOPSIS method for decision making problems under interval-valued intuitionistic fuzzy environment, Applied Mathematical Modelling 35(5): 2544-2556. http://dx.doi.org/10.1016/j.apm.2010.11.025

Shannon, C. E. 1948. A mathematical theory of communication, Bell System Technical Journal 27:379-423. http://dx.doi.org/10.1002/j.1538-7305.1948.tb01338.x

Shang, X. G.; Jiang, W. S. 1997. A note on fuzzy information measures, Pattern Recognition Letters 18(5): 425-432. http://dx.doi.org/10.1016/S0167-8655(97)00028-7

Sugeno, M. 1974. Theory of fuzzy integral and its application: Doctorial Dissertation. Tokyo Institute of Technology.

Tan, C. Q. 2011a. Generalized intuitionistic fuzzy geometric aggregation operator and its application to multi-criteria group decision making, Soft Computing 15(5): 867-876.

http://dx.doi.org/10.1007/s00500-010-0554-6

Tan, C. Q. 2011b. A multi-criteria interval-valued intuitionistic fuzzy group decision making with Choquet integral-based TOPSIS, Expert Systems with Applications 38(4): 3023-3033. http://dx.doi.org/10.1016/j.eswa.2010.08.092

Tan, C. Q.; Chen, X. H. 2010a. Induced Choquet ordered averaging operator and its application to group decision making, International Journal of Intelligent Systems 25(1): 59-82. http://dx.doi.org/10.1002/int.v25:1

Tan, C. Q.; Chen, X. H. 2010b. Intuitionistic fuzzy Choquet integral operator for multi-criteria decision making, Expert Systems with Applications 37(1): 149-157. http://dx.doi.org/10.1016/j.eswa.2009.05.005

Tan, C. Q.; Chen, X. H. 2011. Induced intuitionistic fuzzy Choquet integral operator for multi-criteria decision making, International Journal of Intelligent Systems 26(7): 659-686. http://dx.doi.org/10.1002/int.20489

Tan, C. Q.; Wu, D. S. D.; Ma, B. J. 2011. Group decision making with linguistic preference relations with application to supplier selection, Expert Systems with Applications 38(12): 14382-14389. http://dx.doi.org/10.1016/j.eswa.2011.04.036

Wang, P. 2009. QoS-aware web services selection with intuitionistic fuzzy set under consumer's vague Perception, Expert Systems with Applications 36(3): 4460-4466. http://dx.doi.org/10.1016/j.eswa.2008.05.007

Wang, J. Q.; Li, J. J. 2011. Multi-criteria fuzzy decision-making method based on cross entropy and score functions, Expert Systems with Applications 38(1): 1032-1038. http://dx.doi.org/10.1016/j.eswa.2010.07.137

Xia, M. M.; Xu, Z. S. 2012. Entropy/cross entropy-based group decision making under intuitionistic fuzzy environment, Information Fusion 13(1):31-47. http://dx.doi.org/10.1016/j.inffus.2010.12.001 
Xu, Z. S.; Yager, R. R. 2006. Some geometric aggregation operators based on intuitionistic fuzzy sets, International Journal of General Systems 35(4): 417-433. http://dx.doi.org/10.1080/03081070600574353

$\mathrm{Xu}, \mathrm{Z}$. S. 2007a. Intuitionistic fuzzy aggregation operators, IEEE Transactions on Fuzzy Systems 15(6): 1179-1187. http://dx.doi.org/10.1109/TFUZZ.2006.890678

Xu, Z. S. 2007b. Methods for aggregating interval-valued intuitionistic fuzzy information and their application to decision making, Control and Decision 22(2): 215-219.

$\mathrm{Xu}, \mathrm{Z}$. S. 2007c. Intuitionistic preference relations and their application in group decision making, Information Sciences 177(11): 2363-2379. http://dx.doi.org/10.1016/j.ins.2006.12.019

$\mathrm{Xu}, \mathrm{Z}$. S.; Chen, J. 2007. On geometric aggregation over interval-valued intuitionistic fuzzy information, in Fourth International Conference on Fuzzy Systems and Knowledge Discovery (FSKD), 24-27 August 2007, Haikou, 2: 466-471. http://dx.doi.org/10.1109/FSKD.2007.427

$\mathrm{Xu}, \mathrm{Z}$. S. 2008. Dynamic intuitionistic fuzzy multi-attribute decision making, International Journal of Approximate Reasoning 48(1): 246-262. http://dx.doi.org/10.1016/j.ijar.2007.08.008

$\mathrm{Xu}, \mathrm{Z}$. S.; Chen, J.; Wu, J. J. 2008. Clustering algorithm for intuitionistic fuzzy sets, Information Sciences 178(19): 3775-3790. http://dx.doi.org/10.1016/j.ins.2008.06.008

Xu, Z. S.; Cai, X. Q. 2010a. Recent advances in intuitionistic fuzzy information aggregation, Fuzzy Optimum and Decision Making 9(4): 359-381. http://dx.doi.org/10.1007/s10700-010-9090-1

Xu, Z. S.; Cai, X. Q. 2010b. Nonlinear optimization models for multiple attribute group decision making with intuitionistic fuzzy information, International Journal of Intelligent Systems 25(6): 489-513. http://dx.doi.org/10.1002/int.20409

Xu, Z. S.; Hu, H. 2010. Projection models for intuitionistic fuzzy multiple attribute decision making, International Journal of Information Technology and Decision Making 9(2): 267-280. http://dx.doi.org/10.1142/S0219622010003816

$\mathrm{Xu}, \mathrm{Z}$. S. 2010. Choquet integrals of weighted intuitionistic fuzzy information, Information Sciences 180(5): 726-736. http://dx.doi.org/10.1016/j.ins.2009.11.011

Yager, R. R. 2003. Induced aggregation operators, Fuzzy Sets and Systems 137(1): 59-69. http://dx.doi.org/10.1016/S0165-0114(02)00432-3

Yager, R. R. 2009. Some aspects of intuitionistic fuzzy sets, Fuzzy Optimization and Decision Making 8(1): 67-90. http://dx.doi.org/10.1007/s10700-009-9052-7

Ye, J. 2010. Multicriteria fuzzy decision-making method using entropy weights-based correlation coefficients of interval-valued intuitionistic fuzzy sets, Applied Mathematical Modelling 34(12): 3864-3870. http://dx.doi.org/10.1016/j.apm.2010.03.025

Ye, J. 2011. Fuzzy cross entropy of interval-valued intuitionistic fuzzy sets and its optimal decision-making method based on the weights of alternatives, Expert Systems with Applications 38(5): 6179-6183. http://dx.doi.org/10.1016/j.eswa.2010.11.052

Yue, Z. L. 2011. Deriving decision maker's weights based on distance measure for interval-valued intuitionistic fuzzy group decision making, Expert Systems with Applications 381(9): 1665-11670.

Zadeh, L. A. 1965. Fuzzy sets, Information Control 8(3): 338-353. http://dx.doi.org/10.1016/S0019-9958(65)90241-X

Zhang, H. M.; Yu, L. Y. 2012. MADM method based on cross entropy and extended TOPSIS with intervalvalued intuitionistic fuzzy sets, Knowledge-Based Systems 30(1): 115-120. http://dx.doi.org/10.1016/j.knosys.2012.01.003

Zhang, X. M.; Xu, Z. S.; Yu, X. H. 2011. Shapley value and Choquet integral-based operators for aggregating correlated intuitionistic fuzzy information, Information: An International Interdisciplinary Journal 14(6): 1847-1858. 
Fanyong MENG is an associate professor in School of Management at Qingdao Technological University, Qiangdao, China, and he is a post-doctoral candidate at Central South University, Changsha, China. He received the $\mathrm{PhD}$ degree in School of Management and Economics at Beijing Institute of Technology, Beijing, China. His research interests are in the areas of Optimization theory and methods, game theory and decision making. He has published over 50 research articles in reputed international journals of mathematical and information sciences.

Qiang ZHANG is a professor in School of Management and Economics at Beijing Institute of Technology, Beijing, China. He received the PhD degree in School of Traffic and Transportation at Southwest Jiao Tong University, Chengdu, China. At present, he has contributed over 200 journal articles to reputed international journals of applied mathematics and Information sciences. He is an associate editor of Journal of Intelligent and Fuzzy Systems. His current research interests include management decisions in quantitative theory and method, the modern logistics and supply chain management, uncertain system theory and application.

Jiaquan ZHAN is a lecturer in School of Management at Qingdao Technological University, Qiangdao, China. He received the $\mathrm{PhD}$ degree in School of Management and Economics at Beijing Institute of Technology, Beijing, China. His research interests are in the areas of game theory and decision making. He has published over 10 research articles in reputed international journals of mathematical and information sciences. 